\title{
Automatic Shadow Detection and Removal from a Single Image
}

\author{
S. H. Khan, M. Bennamoun, Member, IEEE, F. Sohel and R. Togneri, Senior Member, IEEE
}

\begin{abstract}
We present a framework to automatically detect and remove shadows in real world scenes from a single image. Previous works on shadow detection put a lot of effort in designing shadow variant and invariant hand-crafted features. In contrast, our framework automatically learns the most relevant features in a supervised manner using multiple convolutional deep neural networks (ConvNets). The features are learned at the super-pixel level and along the dominant boundaries in the image. The predicted posteriors based on the learned features are fed to a conditional random field model to generate smooth shadow masks. Using the detected shadow masks, we propose a Bayesian formulation to accurately extract shadow matte and subsequently remove shadows. The Bayesian formulation is based on a novel model which accurately models the shadow generation process in the umbra and penumbra regions. The model parameters are efficiently estimated using an iterative optimization procedure. Our proposed framework consistently performed better than the state-of-the-art on all major shadow databases collected under a variety of conditions.
\end{abstract}

Index Terms_Feature Learning; Bayesian shadow removal; Conditional Random Field; ConvNets; Shadow detection; Shadow matting

\section{INTRODUCTION}

Shadows are a frequently occurring natural phenomenon, whose detection and manipulation are important in many computer vision (e.g., visual scene understanding) and computer graphics applications. As early as the time of Da Vinci, the properties of shadows were well studied [1]. Recently, shadows have been used for tasks related to object shape [2, 3], size, movement [4], number of light sources and illumination conditions [5]. Shadows have a particular practical importance in augmented reality applications, where the illumination conditions in a scene can be used to seamlessly render virtual objects and their casted shadows. Contrary to the above mentioned assistive roles, shadows can also cause complications in many fundamental computer vision tasks. For instance, they can degrade the performance of object recognition, stereo, shape reconstruction, image segmentation and scene analysis. In digital photography, information about shadows and their removal can help to improve the visual quality of photographs. Shadows are also a serious concern for aerial imaging and object tracking in video sequences [6].

Despite the ambiguities generated by shadows, the Human Visual System (HVS) does not face any real difficulty in filtering out the degradations caused by

- S. H. Khan, M. Bennamoun and F. Sohel are with the School of Computer Science and Software Engineering, The University of Western Australia, 35 Stirling Highway, Crawley, WA 6009, Australia.

E-mail: salman.khan@research.uwa.edu.au, \{mohammed.bennamoun, ferdous.sohel\}@@wa.edu.au

- R. Togneri is with the School of Electrical, Electronic and Computer Engineering, The University of Western Australia, 35 Stirling Highway, Crawley, WA 6009, Australia.

E-mail: roberto.togneri@uwa.edu.au

Manuscript received April 25, 2014; revised April 28, 2015. shadows. We need to equip machines with such visual comprehension abilities. Inspired by the hierarchical architecture of the human visual cortex, many deep representation learning architectures have been proposed in the last decade. We draw our motivation from the recent successes of these deep learning methods in many computer vision tasks where learned features out-performed hand-crafted features [7]. On that basis, we propose to use multiple convolutional neural networks (ConvNets) to learn useful feature representations for the task of shadow detection. ConvNets are biologically inspired deep network architectures based on Hubel and Wiesel's [8] work on the cat's primary visual cortex. Once shadows are detected, an automatic shadow removal algorithm is proposed which encodes the detected information in the likelihood and prior terms of the proposed Bayesian formulation. Our formulation is based on a generalized shadow generation model which models both the umbra and penumbra regions. To the best of our knowledge, we are the first to use 'learned features' in the context of shadow detection, as opposed to the common carefully designed and hand-crafted features. Moreover, the proposed approach detects and removes shadows automatically without any human input (Fig. 1).

Our proposed shadow detection approach combines local information at image patches with the local information across boundaries (Fig. 1). Since the regions and the boundaries exhibit different types of features, we split the detection procedure into two respective portions. Separate ConvNets are consequently trained for patches extracted around the scene boundaries and the super-pixels. Predictions made by the ConvNets are local and we therefore need to exploit the higher level interactions between the neighboring pixels. For this 


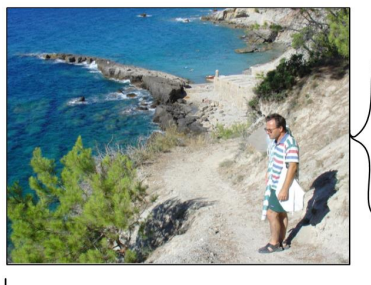

(a)

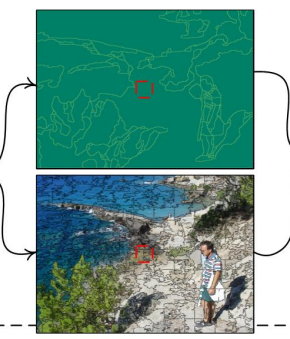

(b)

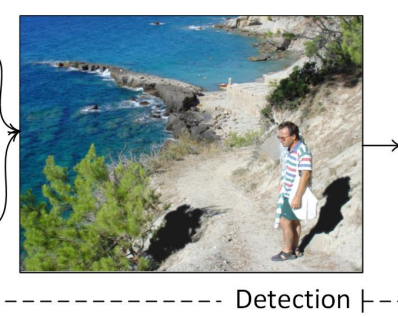

(c)

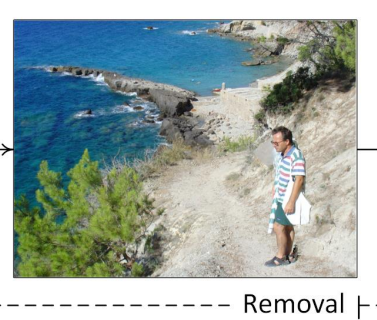

(d)

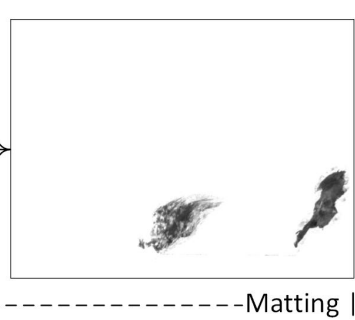

(e)

Fig. 1: From left to right: Original image (a). Our framework first detects shadows (c) using the learned features along the boundaries (top image in (b)) and the regions (bottom image in (b)). It then extracts the shadow matte (e) and removes it to produce a shadow free image $(\mathrm{d})$.

purpose, we incorporate local beliefs in a Conditional Random Field (CRF) model which enforces the labeling consistency over the nodes of a grid graph defined on an image (Sec. 3). This removes isolated and spurious labeling outcomes and encourages neighboring pixels to adopt the same label.

Using the detected shadow mask, we identify the umbra (Latin meaning shadow), penumbra (Latin meaning almost-shadow) and shadow-less regions and propose a Bayesian formulation to automatically remove shadows. We introduce a generalized shadow generation model which separately defines the umbra and penumbra generation process. The resulting optimization problem has a relatively large number of unknown parameters, whose MAP estimates are efficiently computed by alternatively solving for the parameters (Eq. 26). The shadow removal process also extracts smooth shadow matte that can be used in applications such as shadow compositing and editing (Sec. 4).

A preliminary version of this research (which solely focuses on shadow detection) appeared in [9]. In addition, the current study includes: (1) a new approach to estimate shadow statistics, (2) automatic shadow removal and shadow matte extraction, (3) a substantial number of additional experiments, analysis and limitations, (4) possible applications in many computer vision and graphics tasks.

\section{Related Work and Contributions}

Shadow Detection: One of the most popular methods to detect shadows is to use a variety of shadow variant and invariant cues to capture the statistical and deterministic characteristics of shadows [10, 11, 12, 13, 14]. The extracted features model the chromatic, textural $[10,11,13,14]$ and illumination [12, 15] properties of shadows to determine the illumination conditions in the scene. Some works give more importance to features computed across image boundaries, such as intensity and color ratios across boundaries and the computation of texton features on both sides of the edges [11, 16]. Although these feature representations are useful, they are based on assumptions that may not hold true in all cases. As an example, chromatic cues assume that the texture of the image regions remains the same across shadow boundaries and only the illumination is different. This approach fails when the image regions under shadows are barely visible. Moreover, all of these methods involve a considerable effort in the design of hand-crafted features for shadow detection and feature selection (e.g., the use of ensemble learning methods to rank the best features $[10,11])$. Our data-driven framework is different and unique: we propose to use deep feature learning methods to 'learn the most relevant features' for shadow detection.

Owing to the challenging nature of the shadow detection problem, many simplistic assumptions are commonly adopted. Previous works made assumptions related to the illumination sources [5], the geometry of the objects casting shadows and the material properties of the surfaces on which shadows are cast. For example, Salvador et al. [14] consider object cast shadows while Lalonde et al. [11] only detect shadows that lie on the ground. Some methods use synthetically generated training data to detect shadows [17]. Techniques targeted for video surveillance applications take advantage of multiple images [18] or time-lapse sequences $[19,20]$ to detect shadows. User assistance is also required by many proposed techniques to achieve their attained performances [21, 22]. In contrast, our shadow detection method makes absolutely 'no prior assumptions' about the scene, the shadow properties, the shape of objects, the image capturing conditions and the surrounding environments. Based on this premise, we tested our proposed framework on all of the publicly available databases for shadow detection from single images. These databases contain common real world scenes with artifacts such as noise, compression and color balancing effects.

Shadow Removal and Matting: Almost all approaches that are employed to either edit or remove shadows are based on models that are derived from the image formation process. A popular choice is to physically model the image into a decomposition of its intrinsic images along with some parameters that are responsible for the generation of shadows. As a result, the shadow removal process is reduced to the estimation of the model parameters. Finlayson et al. [23, 24] addressed this problem by nullifying the shadow edges and reintegrating the image, 
which results in the estimation of the additive scaling factor. Since such global integration (which requires the solution of a 2D Poisson equation [23, 25]) causes artifacts, the integration along a 1D Hamiltonian path [26] is proposed for shadow removal. However, these and other gradient based methods (such as [27, 28]) do not account for the shadow variations inside the umbra region. To address this shortcoming, Arbel and Hel-Or [29] treat the illumination recovery problem as a $3 \mathrm{D}$ surface reconstruction and use a thin plate model to successfully remove shadows lying on curved surfaces. Alternatively, information theory based techniques are proposed in $[25,30]$ and a bilateral filtering based approach is recently proposed in [31] to recover intrinsic (illumination and reflectance) images. However, these approaches either require user assistance, calibrated imaging sensors, careful parameter selection or considerable processing times. To overcome these shortcomings, some reasonably fast and accurate approaches have been proposed which aim to transfer the color statistics from the nonshadow regions to the shadow regions ('color transfer based approaches' e.g., [21, 32, 33, 34, 35]). Our proposed shadow removal algorithm also belongs to the category of color transfer based approaches. However, in contrast to previous related works, we propose a generalized image formation model which enables us to deal with non-uniform umbra regions as well as soft shadows. Color transfer is also made at multiple spatial levels, which helps in the reduction of noise and color artifacts. An added advantage of our approach is our ability to separate smooth shadow matte from the actual image.

Several assumptions are made in the shadow removal literature due to the ill-posed nature of recovering the model parameters for each pixel. The camera sensor parameters are needed in [23, 31]. Multiple narrowband sensor outputs for each scene are required in [31], while [2] employs a sequence of images to recover the intrinsic components. Lambertian surface and Planckian lightening assumptions are made in [31]. Though several approaches work just on a single image, they require considerable user interaction to identify either tri-maps [36], quad-maps [33, 34], gradients [37] or exact shadow boundaries [27, 28]. Su and Chen [38] tried to minimize the user effort by specifying the complete shadow boundary from the user provided strokes. In contrast, our framework does not require any form of user interaction and makes no assumption regarding the camera or scene properties (except that the object surfaces are assumed to be Lambertian).

The key contributions of our work are outlined below:

- We propose a new approach for robust shadow detection combining both regional and acrossboundary learned features in a probabilistic framework involving CRFs (Sec. 3).

- Our proposed method automatically learns the most relevant feature representations from raw pixel values using multiple ConvNets (Sec. 3).

- We propose a generalized shadow formation model along with automatic color statistics modeling using only detected shadow masks (Sec. 4.1 and 4.2).

- Our proposed Bayesian formulation for the shadow removal problem integrates multi-level color transfer and the resulting cost function is efficiently optimized to give superior results (Sec. 4.3 and 4.4).

- We performed extensive quantitative evaluation to prove that the proposed framework is robust, lessconstrained and generalisable across different types of scenes (Sec. 5).

\section{Proposed Shadow Detection Frame- WORK}

Given a single color image, we aim to detect and localize shadows precisely at the pixel level (see block diagram in Fig. 2). If $\mathbf{y}$ denotes the desired binary mask encoding class relationships, we can model the shadow detection problem as a conditional distribution:

$$
\mathcal{P}(\mathbf{y} \mid \mathbf{x} ; \mathbf{w})=\frac{1}{Z(\mathbf{w})} \exp (-\mathrm{E}(\mathbf{y}, \mathbf{x} ; \mathbf{w}))
$$

where, the parameter vector $\mathrm{w}$ includes the weights of the model, the manifest variables are represented by $\mathbf{x}$ where $\mathbf{x}_{i}$ denotes the intensity of pixel $i \in\left\{p_{i}\right\}_{1 \times N}$ and $Z(\mathbf{w})$ denotes the partition function. The energy function is composed of two potentials; the unary potential $\psi_{i}$ and the pairwise potential $\psi_{i j}$ :

$$
\mathrm{E}(\mathbf{y}, \mathbf{x} ; \mathbf{w})=\sum_{i \in \mathcal{V}} \psi_{i}\left(y_{i}, \mathbf{x} ; \mathbf{w}_{i}\right)+\sum_{(i, j) \in \mathcal{E}} \psi_{i j}\left(y_{i j}, \mathbf{x} ; \mathbf{w}_{i j}\right)
$$

In the following discussion, we will explain how we model these potentials in a CRF framework.

\subsection{Feature Learning for Unary Predictions}

The unary potential in Eq. 2 considers the shadow properties both at the regions and at the boundaries inside an image.

$$
\psi_{i}\left(y_{i}, \mathbf{x} ; \mathbf{w}_{i}\right)=\overbrace{\phi_{i}^{r}\left(y_{i}, \mathbf{x} ; \mathbf{w}_{i}^{r}\right)}^{\text {region }}+\overbrace{\phi_{i}^{b}\left(y_{i}, \mathbf{x} ; \mathbf{w}_{i}^{b}\right)}^{\text {boundary }}
$$

We define each of the boundary and regional potentials, $\phi^{r}$ and $\phi^{b}$ respectively, in terms of probability estimates from the two separate ConvNets,

$$
\begin{aligned}
& \phi_{i}^{r}\left(y_{i}, \mathbf{x} ; \mathbf{w}_{i}^{r}\right)=-\mathbf{w}_{i}^{r} \log \mathcal{P}_{\mathrm{cnn} 1}\left(y_{i} \mid \mathbf{x}_{\mathbf{r}}\right) \\
& \phi_{i}^{b}\left(y_{i}, \mathbf{x} ; \mathbf{w}_{i}^{b}\right)=-\mathbf{w}_{i}^{b} \log \mathcal{P}_{\text {cnn2 } 2}\left(y_{i} \mid \mathbf{x}_{\mathrm{b}}\right)
\end{aligned}
$$

This is logical because the features to be estimated at the boundaries are likely to be different from the ones estimated inside the shadowed regions. Therefore, we train two separate ConvNets, one for the regional potentials and the other for the boundary potentials.

The ConvNet architecture used for feature learning consists of alternating convolution and sub-sampling layers (Fig. 3). Each convolutional layer in a ConvNet consists of filter banks which are convolved with the input feature maps. The sub-sampling layers pool the incoming features to derive invariant representations. This 


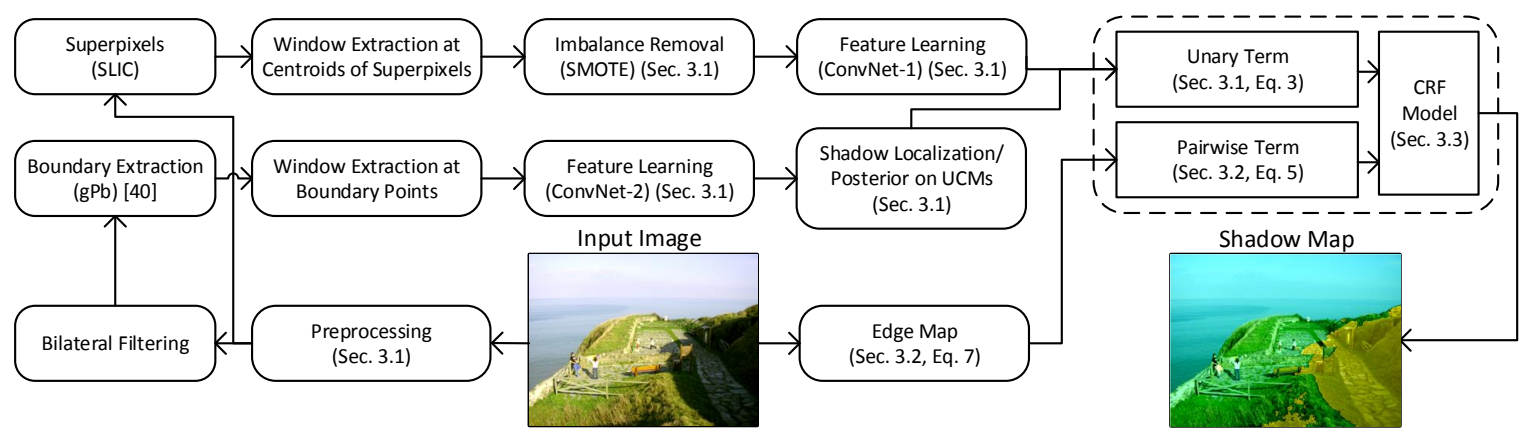

Fig. 2: The proposed shadow detection framework. (Best viewed in color)

layered structure enables ConvNets to learn multilevel hierarchies of features. The final layer of the network is fully connected and comes just before the output layer. This layer works as a traditional MLP with one hidden layer followed by a logistic regression output layer which provides a distribution over the classes. Overall, after the network has been trained, it takes an RGB patch as an input and processes it to give a posterior distribution over binary classes.

ConvNets operate on equi-sized windows, so it is required to extract patches around desired points of interest. For the case of regional potentials, we extract super-pixels by clustering the homogeneous pixels ${ }^{1}$. Afterwards, a patch $\left(\mathcal{I}^{r}\right)$ is extracted by centering a $\tau_{s} \times \tau_{s}$ window at the centroid of each superpixel. Similarly for boundary potentials, we first apply a Bilateral filter and then extract boundaries using the $\mathrm{gPb}$ technique [40]. We traverse each boundary with a stride $\lambda_{b}$ and extract a $\tau_{s} \times \tau_{s}$ patch at each step to incorporate local context ${ }^{2}$. Therefore, ConvNets operate on sets of boundary and super-pixel patches, $\mathbf{x}_{\mathrm{r}}=\left\{\mathcal{I}^{r}(i, j)\right\}_{1 \times\left|\mathcal{F}_{\text {slic }}(\mathbf{x})\right|}$ and $\mathbf{x}_{\mathrm{b}}=\left\{\mathcal{I}^{b}(i, j)\right\}_{1 \times \frac{\left|\mathcal{F}_{\mathrm{gpb}}(\mathbf{x})\right|}{\lambda_{\mathrm{b}}}}$ respectively, where $|$.$| is$ the cardinality operator. Note that we include synthetic data (generated by artificial linear transformations [41]) during the training process. This data augmentation is important not only because it removes the skewed class distribution of the shadowed regions but it also results in an enhanced performance. Moreover, data augmentation helps to reduce the overfitting problem in ConvNets (e.g., in [42]) which results in the learning of more robust feature representations.

During the training process, we use stochastic gradient descent to automatically learn feature representations in a supervised manner. The gradients are computed using back-propagation to minimize the cross entropy loss function [43]. We set the training parameters (e.g., momentum and weight decay) using a cross validation process. The training samples are shuffled randomly before training since the network can learn faster from unexpected samples. The weights of the ConvNet were initialized with randomly drawn samples from a Gaussian distribution of zero mean and a variance that is

1. In our implementation we used SLIC [39], due to its efficiency.

2. the step size is $\lambda_{b}=\tau_{s} / 4$ to get partially overlapping windows.

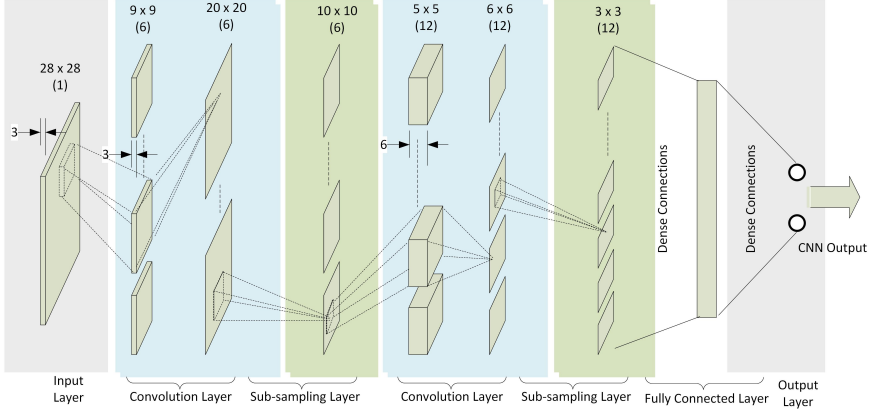

Fig. 3: ConvNet Architecture used for Automatic Feature Learning to Detect Shadows.

inversely proportional to the fan-in measure of neurons. The number of epochs during the training of ConvNets is set by an early stopping criterion based on a small validation set. The initial learning rate is heuristically chosen by selecting the largest rate which resulted in the convergence of the training error. This rate is decremented by a factor of $v=0.5$ after every 20 epochs.

The ConvNet trained on boundary patches learn to separate shadow and reflectance edges while the ConvNet trained on regions can differentiate between shadow and non-shadow patches. For the case of the regions, the posteriors predicted by ConvNet are assigned to each super pixel in an image. However, for the boundaries, we first localize the probable shadow location using the local contrast and then average the predicted probabilities over each contour generated by the Ultra-metric Contour Maps (UCM) [40].

\subsection{Contrast Sensitive Pairwise Potential}

The pairwise potential in Eq. 2 is defined as a combination of the class transition potential $\phi_{p_{1}}$ and the spatial transition potential $\phi_{p_{2}}$ :

$$
\psi_{i j}\left(y_{i j}, \mathbf{x} ; \mathbf{w}_{i j}\right)=\mathbf{w}_{i j} \phi_{p_{1}}\left(y_{i}, y_{j}\right) \phi_{p_{2}}(\mathbf{x}) .
$$

The class transition potential takes the form of an Ising prior:

$$
\phi_{p_{1}}\left(y_{i}, y_{j}\right)=\alpha \mathbf{1}_{y_{i} \neq y_{j}}= \begin{cases}0 & \text { if } y_{i}=y_{j} \\ \alpha & \text { otherwise }\end{cases}
$$




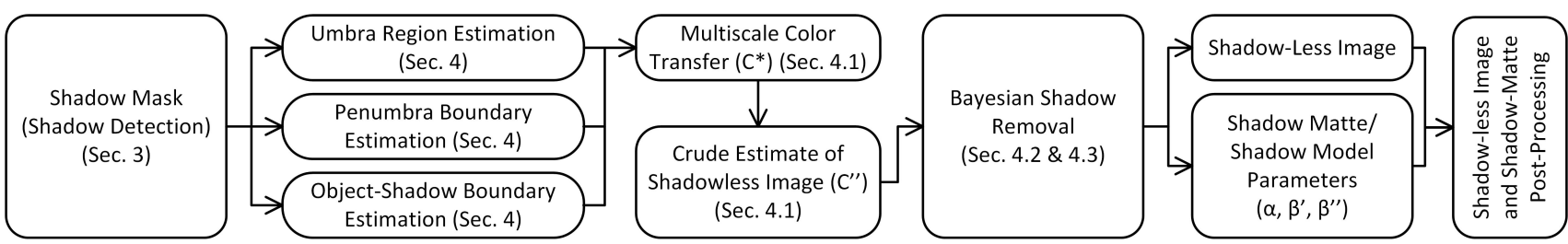

Fig. 4: The Proposed Shadow Removal Framework: After the detection of the shadows in the image, we estimate the umbra, penumbra and object-shadow boundary. Given this information, a multi-level color transfer is applied to obtain a crude estimate of shadow-less image. This rough estimate is further improved using the proposed Bayesian formulation which estimates the optimal shadow-less image along with the shadow model parameters.

The spatial transition potential captures the differences in the adjacent pixel intensities:

$$
\phi_{p_{2}}(\mathbf{x})=\left[\exp \left(-\frac{\left\|x_{i}-x_{j}\right\|^{2}}{\beta_{x}\left\langle\left\|x_{i}-x_{j}\right\|^{2}\right\rangle}\right)\right]
$$

where, $\langle\cdot\rangle$ denotes the average contrast in an image. The parameters $\alpha$ and $\beta_{x}$ were derived using cross validation on each database.

\subsection{Shadow Contour Generation using CRF Model}

We model the shadow contour generation in the form of a two-class scene parsing problem where each pixel is labeled either as a shadow or a non-shadow. This binary classification problem takes probability estimates from the supervised feature learning algorithm and incorporates them in a CRF model. The CRF model is defined on a grid structured graph topology, where graph nodes correspond to image pixels (Eq. 2). When making an inference, the most likely labeling is found using the Maximum a Posteriori (MAP) estimate $\left(\mathbf{y}^{*}\right)$ upon a set of random variables $\mathbf{y} \in \mathcal{L}^{N}$. This estimation turns out to be an energy minimization problem since the partition function $Z(\mathbf{w})$ does not depend on $\mathbf{y}$ :

$$
\mathbf{y}^{*}=\underset{\mathbf{y} \in \mathcal{L}^{N}}{\operatorname{argmax}} \mathcal{P}(\mathbf{y} \mid \mathbf{x} ; \mathbf{w})=\underset{\mathbf{y} \in \mathcal{L}^{N}}{\operatorname{argmin}} \mathrm{E}(\mathbf{y}, \mathbf{x} ; \mathbf{w})
$$

The CRF model proved to be an elegant source to enforce label consistency and the local smoothness over the pixels. However, the size of the training space (labeled images) makes it intractable to compute the gradient of the likelihood. Therefore the parameters of the CRF cannot be found by simply maximizing the likelihood of the hand labeled shadows. Hence, we use the 'margin rescaled algorithm' to learn the parameters (w in Eq. 8) of our proposed CRF model (see Fig 3 in [44] for details). Because our proposed energies are sub-modular, we use graph-cuts for making efficient inferences [45]. In the next section, we describe the details of our shadow removal and matting framework.

\section{Proposed Shadow Removal and Mat- TING FRAMEWORK}

Based on the detected shadows in the image, we propose a novel automatic shadow removal approach. A block diagram of the proposed approach is presented in Fig. 4. The first step is to identify the umbra, penumbra and

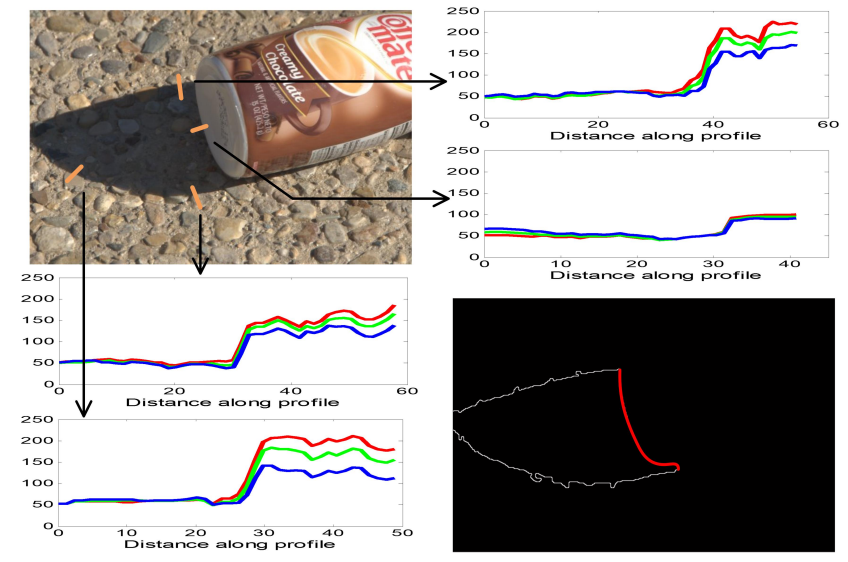

Fig. 5: Detection of Object and Shadow Boundary: We use the gradient profile along the direction perpendicular to a boundary point (four sample profiles are plotted on the anti-diagonal of above figure) to separate the object-shadow boundary (shown in red in lower right image).

the corresponding non-shadowed regions in an image. We also need to identify the boundary where the actual object and its shadow meet. This identification helps to avoid any errors during the estimation of shadow/nonshadow statistics (e.g., color distribution). In previous works (such as [21, 29, 34]), this process has been carried out manually through human interaction. We, however, propose a simple procedure to automatically estimate the umbra, penumbra regions and the object-shadow boundary.

Heuristically, the object-shadow boundary is relatively darker compared to other shadow boundaries where differences in light intensity are significant. Therefore, given a shadow mask, we calculate the boundary normals at each point. We cluster the boundary points according to the direction of their normals. This results in separate boundary segments which join to form the boundary contour around the shadow. Then, the boundary segments in the shadow contour with a minimum relative change in intensity are classified to represent the object-shadow boundary. If $\varrho_{b}^{c}$ denotes the mean intensity change along the normal direction at a boundary segment $b$ of the shadow contour $c$, all boundary segments s.t. $\varrho_{b}^{c} / \varrho_{\max }^{c} \leq 0.5$ are considered to correspond to the segments which separate the object and its cast shadow. This simple procedure performs reasonably well for most of our test examples (Fig. 5). In the case where 


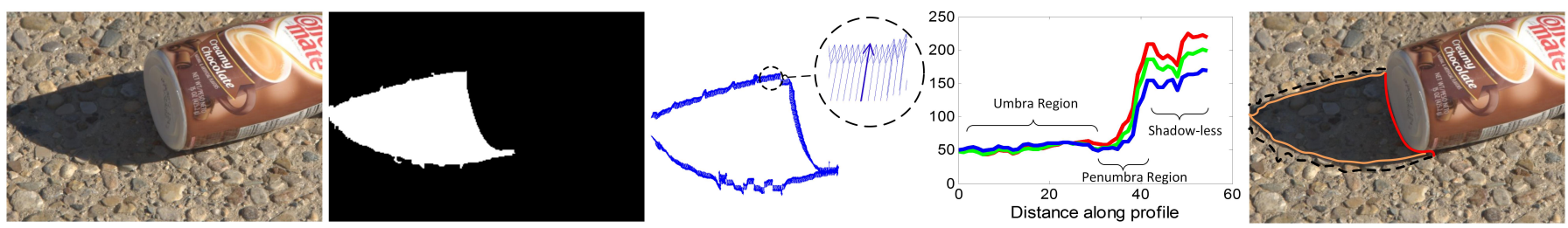

Fig. 6: Detection of Umbra and Penumbra Regions: With the detected shadow map ( $2^{\text {nd }}$ image from left), we estimate the umbra and penumbra regions (rightmost image) by analyzing the gradient profile $\left(4^{\text {th }}\right.$ image from left) at the boundary points.

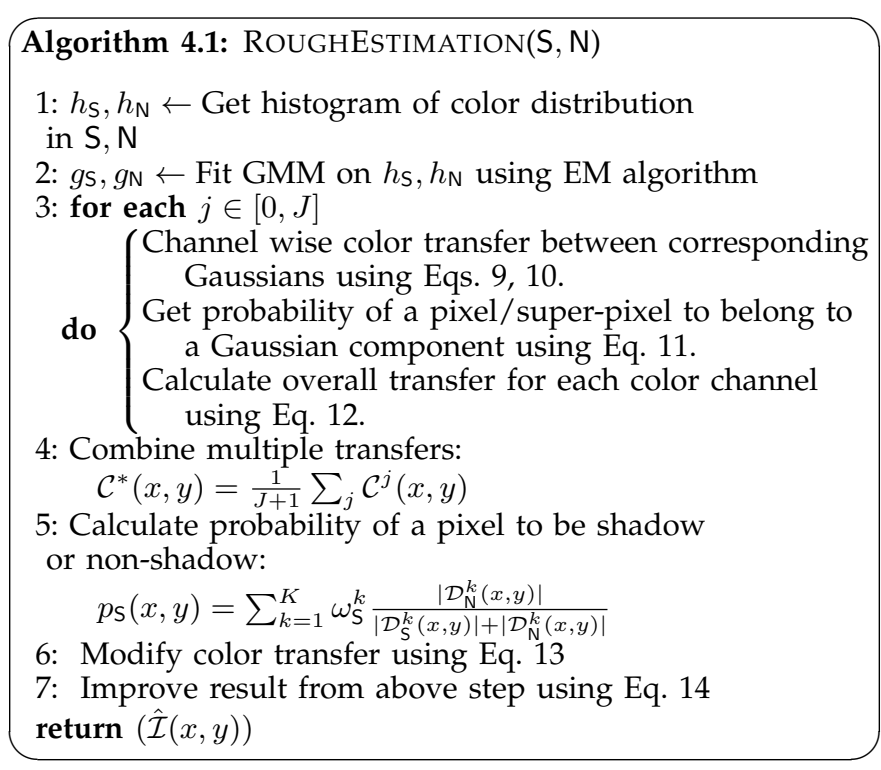

the object shadow boundary is not visible, no boundary portion is classified as an object shadow boundary and the shadow-less statistics are taken from all around the shadow region. In most cases, this does not affect the removal performance as long as the object-shadow boundary is not very large compared to the total shadow boundary.

To estimate the umbra and penumbra regions, the boundary is estimated at each point of the shadow contour by fitting a curve and finding the corresponding normal direction. This procedure is adopted to extract accurate boundary estimates instead of local normals which can result in erroneous outputs at times. We propagate the boundaries along the estimated normal directions until the intensity change becomes insignificant (Fig. 6). This results in an approximation of the penumbra region. We then exclude this region from the shadow mask and the remaining region is considered as the umbra region. The region immediately adjacent to the shadow region, with twice the width of the penumbra region is treated as the non-shadow region. Note that our approach is based on the assumption that the texture remains approximately the same across the shadow boundary.

\subsection{Rough Estimation of Shadow-less Image by Color-transfer}

The rough shadow-less image estimation process is based on the one adopted by the color transfer techniques in [32] and [34]. As opposed to [32, 34], we perform a multilevel color transfer and our method does not require any user input. The color statistics of the shadowed as well as the non-shadowed regions are modeled using a Gaussian mixture model (GMM). For this purpose, a continuous probability distribution function is estimated from the histograms of both regions using the Expectation-Maximization (EM) algorithm. The EM algorithm is initialized using an unsupervised clustering algorithm (k-means in our implementation) and the EM iterations are carried out until convergence. We treat each of the R, G and B channels separately and fit mixture models to each of the respective histograms. It is considered that the estimated Gaussians, in the shadow and non-shadow regions, correspond to each other when arranged according to their means. Therefore, the color transfer is computed among the corresponding Gaussians using the following pair of equations:

$$
\begin{gathered}
\mathcal{D}_{\mathrm{S}}^{k}(x, y)=\frac{\mathcal{I}(x, y)-\mu_{\mathrm{S}}^{k}}{\sigma_{\mathrm{S}}^{k}} \\
\mathcal{C}^{k}(x, y)=\mu_{\mathrm{N}}^{k}+\sigma_{\mathrm{N}}^{k} \mathcal{D}_{\mathrm{S}}(x, y)
\end{gathered}
$$

where $\mathcal{D}(\cdot)$ measures the normalized deviation for each pixel, $\mathrm{S}$ and $\mathrm{N}$ denote the shadow and non-shadow regions respectively. The index $k$ is in range $[1, K]$, where $K$ denotes the total number of Gaussians used to approximate the histogram of S. The probability that a pixel (with coordinates $x, y$ ) belongs to a certain Gaussian component can be represented in terms of its normalized deviation:

$$
p_{G}^{k}(x, y)=\left(\left|\mathcal{D}_{\mathrm{S}}^{k}(x, y)\right| \sum_{k=1}^{K} \frac{1}{\left|\mathcal{D}_{\mathrm{S}}^{k}(x, y)\right|}+\epsilon\right)^{-1}
$$

The overall transfer is calculated by taking the weighted sum of transfers for all Gaussian components:

$$
\mathcal{C}^{j=0}(x, y)=\sum_{k=1}^{K} p_{G}^{k}(x, y) \mathcal{C}^{k}(x, y) .
$$

The color transfer performed at each pixel location (i.e. at level $j=0$ ) using Eq. 12 is local, and it thus, does not accurately restore the image contrast in the shadowed regions. Moreover, this local color transfer is prone to noise and discontinuities in illumination. We 
therefore resort to a hierarchical strategy which restores color at multiple levels and combines all transfers which results in a better estimation of the shadow-less image. A graph based segmentation procedure [46] is used to group the pixels. This clustering is performed at $J$ levels, which we set to 4 in the current work based on the performance on a small validation set, where we noted an over-smoothing and a low computational efficiency when $J \geq 5$. Since, the segment size is kept quite small, it is highly unlikely that the differently colored pixels will be grouped together. At each level $j \in[1, J]$, the mean of each cluster is used in the color transfer process (using Eqs. 9, 10) and the resulting estimate (Eq. 12) is distributed to all pixels in the cluster. This gives multiple color transfers $\mathcal{C}^{j}(x, y)$ at $J$ different resolutions plus the local color transfer i.e. $\mathcal{C}^{j=0}(x, y)$. At each level, a pixel or a super-pixel is treated as a discrete unit during the color transfer process. The resulting transfers are integrated to produce the final outcome: $\mathcal{C}^{*}(x, y)=\frac{1}{J+1} \sum_{j=0}^{J} \mathcal{C}^{j}(x, y)$. This process helps in reducing the noise. It also restores a better texture and improves the quality of the restored image. It should be noted that our hierarchical strategy helps in successfully retaining the self shading patterns in the recovered image compared to previous works (Sec. 5.3).

To avoid possible errors due to the small non-shadow regions that may be present in the selected shadow region $S$, we calculate the probability of a pixel to be shadowed using: $p_{\mathrm{S}}(x, y)=\sum_{k=1}^{K} \omega_{\mathrm{S}}^{k} p_{\mathrm{S}}^{k}(x, y)$, where $\omega_{\mathrm{S}}^{k}$ is the weight of Gaussians (learned by the EM algorithm) and $p_{\mathrm{S}}^{k}(x, y)=\left|\mathcal{D}_{\mathrm{N}}^{k}\right| /\left(\left|\mathcal{D}_{\mathrm{S}}^{k}\right|+\left|\mathcal{D}_{\mathrm{N}}^{k}\right|\right)$. The color transfer is modified as:

$$
\mathcal{C}^{\prime}(x, y)=\left(1-p_{\mathrm{S}}(x, y)\right) \mathcal{I}_{\mathbf{S}}(x, y)+p_{\mathrm{S}}(x, y) \mathcal{C}^{*}(x, y)
$$

However, the penumbra region pixels will not get accurate intensity values. To correct this anomaly, we define a relation which measures the probability (in a naive sense) of a pixel to belong to the penumbra region. Since the penumbra region occurs around the shadow boundary, we define it as: $b_{\mathrm{s}}(x, y)=d(x, y) / d_{\max }$. The penumbra region is recovered using the exemplar based inpainting approach of Criminisi et al. [47]. The resulting improved approximation of the shadow-less image is,

$$
\hat{\mathcal{I}}(x, y)=\left(1-b_{\mathbf{S}}(x, y)\right) \mathcal{E}(x, y)+b_{\mathbf{S}}(x, y) \mathcal{C}^{\prime}(x, y)
$$

where, $\mathcal{E}$ is the inpainted image.

In our approach, the crude estimate of a shadowless image (Eq. 14) is further improved using Bayesian estimation (Sec. 4.3). But first we need to introduce the proposed shadow generation model used in our Bayesian formulation (Sec. 4.2).

\subsection{Generalised Shadow Generation Model}

Unlike previous works (such as [13, 21, 27, 34, 35]), which do not differentiate between the umbra and the penumbra regions during the shadow formation process, we propose a model which treats both types of shadow

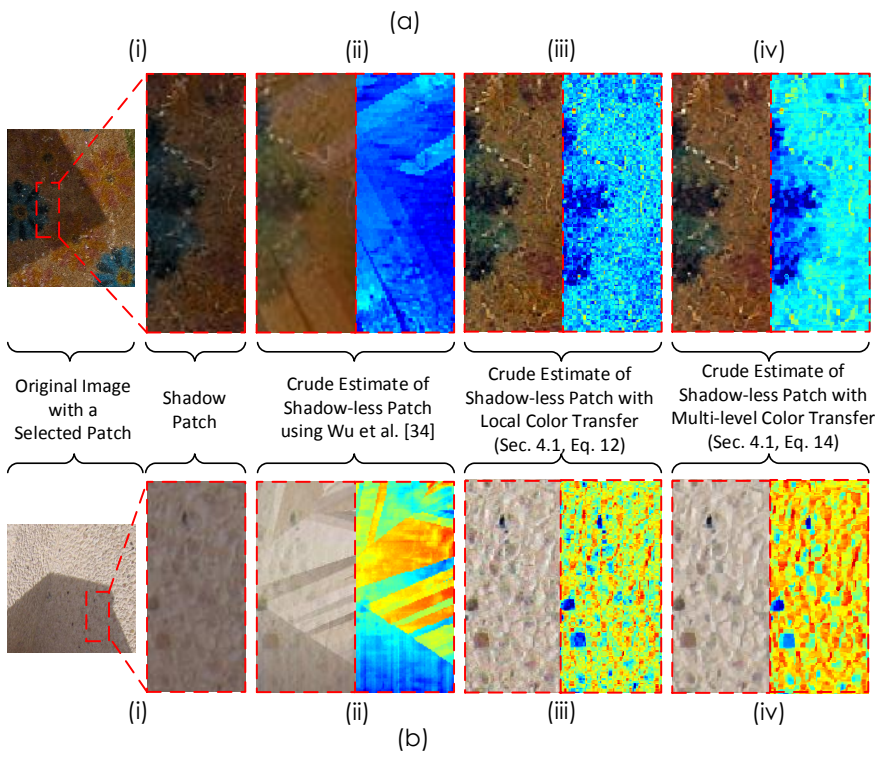

Fig. 7: Multi-level Color Transfer: (from left to right) (i) Two example images ( $a$ and $b$ ), with selected shadow regions. (ii) The recovered shadow-less patch using the technique of $\mathrm{Wu}$ et al. [33]. To highlight the difference with the original patch, we also show the difference image in color. (iii) The result of the local color transfer and its difference with the original patch. (iv) The result of the multi-level color transfer. Note that the multi-level transfer removes noise and preserves the local texture.

regions separately. It is important to make such distinction because the umbra and penumbra regions exhibit distinct illumination characteristics and have a different influence from the direct and indirect light (Fig. 6).

Let us suppose that we have a scene with illuminated and shadowed regions. A normal illuminated image can be represented in terms of two intrinsic images according to the image formation model of Barrow et al. [48]:

$$
\mathcal{I}(x, y)=\mathcal{L}(x, y) \mathcal{R}(x, y)
$$

where $\mathcal{L}$ and $\mathcal{R}$ are the illumination and reflectance respectively and $x, y$ denote the pixel coordinates. The illumination intrinsic image takes into account the illumination differences such as shadows and shading. We assume that a single source of light is casting the shadows. The ambient light is assumed to be uniformly distributed in the environment due to the indirect illumination caused by reflections. Therefore,

$$
\mathcal{I}(x, y)=\left(\mathcal{L}_{d}(x, y)+\mathcal{L}_{i}(x, y)\right) \mathcal{R}(x, y)
$$

A cast shadow is formed when the direct illumination is blocked by some obstructing object resulting in an occlusion. A cast shadow can be described as the combination of two regions created by two distinct phenomena, umbra $(\mathrm{U})$ and penumbra $(\mathrm{P})$. Umbra is surrounded by the penumbra region where the light intensity changes sharply from dark to illuminated. The occlusion which casts the shadow block all of the direct illumination and parts of the indirect illumination to create the umbra 


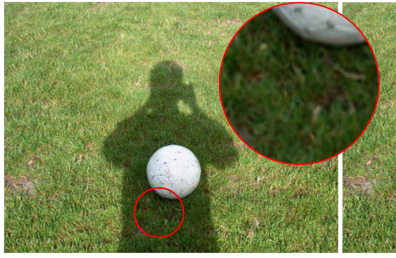

(i)

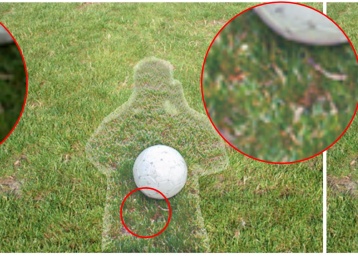

(ii)

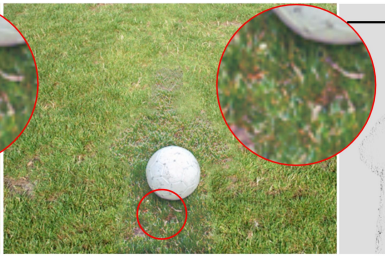

(iii)

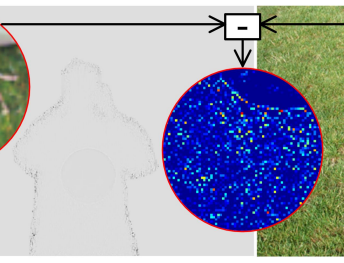

(iv)

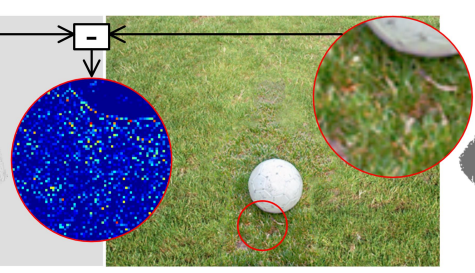

( $v)$

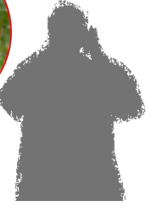

(vi)

Fig. 8: Shadow Removal Steps: (from left to right) (i) An original image with shadow. (ii) An initial estimate of the shadow-less image using a multi-level color transfer strategy. (iii) Improved estimate along the boundaries using in-painting. (iv, v and vi) The Bayesian formulation is optimized to solve for $\alpha$ (iv) and $\beta$ matte (vi) and the final shadow-less image (v).

region. We can represent this as;

$$
\begin{aligned}
\mathcal{I}_{u}(x, y)= & \beta^{\prime}(x, y) \mathcal{L}_{i}(x, y) \mathcal{R}(x, y) \quad \forall x, y \in \mathrm{U} \\
& \because \mathcal{L}_{d}(x, y) \approx 0 \quad \forall x, y \in \mathrm{U}
\end{aligned}
$$

where, $\beta^{\prime}(x, y)$ is the scaling factor for the $\mathrm{U}$ region. Using Eq. 16 and 17, we have;

$$
\begin{gathered}
\mathcal{I}(x, y)=\frac{\mathcal{I}_{u}(x, y)}{\beta^{\prime}(x, y)}+\alpha(x, y) \\
\mathcal{I}_{u}=\mathcal{I}(x, y) \beta^{\prime}(x, y)-\alpha(x, y) \beta^{\prime}(x, y)
\end{gathered}
$$

where, $\alpha(x, y)=\mathcal{L}_{d}(x, y) \mathcal{R}(x, y)$.

For the case of the penumbra region, all direct light is not blocked, rather its intensity decreases from a fully lit region towards the umbra region. Since the major source of change is the direct light, we can neglect the variation caused by the indirect illumination in the penumbra region. Therefore,

$$
\begin{aligned}
\mathcal{I}_{p}(x, y)= & \left(\beta^{\prime \prime}(x, y) \mathcal{L}_{d}(x, y)+\mathcal{L}_{i}(x, y)\right) \mathcal{R}(x, y) \\
& \because \Delta \mathcal{L}_{i}(x, y) \approx 0 \quad \forall x, y \in \mathrm{P}
\end{aligned}
$$

where, $\beta^{\prime \prime}(x, y)$ is the scaling factor for the $\mathrm{P}$ region. Using Eq. 16 and 20, we have:

$$
\mathcal{I}_{p}(x, y)=\mathcal{I}(x, y)-\alpha(x, y)\left(1-\beta^{\prime \prime}(x, y)\right) .
$$

\subsection{Bayesian Shadow Removal and Matting}

Having formulated the shadow generation model, we can now describe the estimation procedure of the model parameters in probabilistic terms. We represent our problem in a well-defined Bayesian formulation and estimate the required parameters using maximum a posteriori estimate (MAP):

$$
\begin{gathered}
\left\{\alpha^{*}, \beta^{*}\right\}=\underset{\alpha, \beta}{\operatorname{argmax}} \mathcal{P}(\alpha, \beta \mid \mathrm{U}, \mathrm{P}, \mathrm{N}) \\
=\underset{\alpha, \beta}{\operatorname{argmax}} \frac{\mathcal{P}(\mathrm{U}, \mathrm{P}, \mathrm{N} \mid \alpha, \beta) \mathcal{P}(\alpha) \mathcal{P}(\beta)}{\mathcal{P}(\mathrm{U}, \mathrm{P}, \mathrm{N})} \\
=\underset{\alpha, \beta}{\operatorname{argmax}} \mathcal{P}_{\ell}(\mathrm{U}, \mathrm{P}, \mathrm{N} \mid \alpha, \beta)+\mathcal{P}_{\ell}(\alpha)+\mathcal{P}_{\ell}(\beta)-\mathcal{P}_{\ell}(\mathrm{U}, \mathrm{P}, \mathrm{N})
\end{gathered}
$$

where, $\mathcal{P}_{\ell}=\log \mathcal{P}(\cdot)$ is the $\log$ likelihood and $\mathrm{U}, \mathrm{P}$ and $\mathrm{N}$ represent the umbra, penumbra and non-shadow regions respectively. The last term in the above equation can be neglected during optimization because it is independent of the model parameters. Therefore:

$$
=\underset{\alpha, \beta}{\operatorname{argmax}} \mathcal{P}_{\ell}(\mathrm{U}, \mathrm{P}, \mathrm{N} \mid \alpha, \beta)+\mathcal{P}_{\ell}(\alpha)+\mathcal{P}_{\ell}(\beta)
$$

Let $\mathcal{I}_{s}(x, y) \quad \forall x, y \in\{\mathrm{U} \cup \mathrm{P}\}$ represent the complete shadow region. Then, the first term in Eq. 25 can be written as a function of $\mathcal{I}_{s}$ since the parameters $\alpha$ and $\beta$ do not affect the region $\mathrm{N}$, therefore:

$$
=\underset{\alpha, \beta}{\operatorname{argmax}} \mathcal{P}_{\ell}\left(\mathcal{I}_{s} \mid \alpha, \beta\right)+\mathcal{P}_{\ell}(\alpha)+\mathcal{P}_{\ell}(\beta)
$$

The first term in Eq. 26 can be modeled by the difference between the current pixel values and the estimated pixel values, as follows:

$$
\begin{aligned}
\mathcal{P}_{\ell}\left(\mathcal{I}_{s} \mid \alpha, \beta\right)=- & \sum_{\{x, y\} \in \mathrm{S}} \frac{\left|\mathcal{I}_{s}(x, y)-\hat{\mathcal{I}}_{s}(x, y)\right|^{2}}{2 \sigma_{\mathcal{I}_{s}}^{2}} \\
- & \sum_{\{x, y\} \in S} \frac{\pi(x, y) \eta(x, y)|\mathcal{I}(x, y)-\hat{\mathcal{I}}(x, y)|^{2}}{2 \sigma_{\mathcal{I}}^{2}}
\end{aligned}
$$

where, $\eta(x, y)=1-\frac{\lambda(x, y)}{\lambda_{\max }}$ and $\pi$ is an indicator function which switches on for the penumbra region pixels. $\lambda(\cdot)$ is the distance metric which quantifies the shortest distance between a valid shadow boundary (i.e., excluding the object-shadow boundary). The estimated shadowed image $\left(\hat{\mathcal{I}}_{s}\right)$ can be decomposed as follows using Eqs. 19 and 21.

$\hat{\mathcal{I}}_{s}(x, y)= \begin{cases}(\hat{\mathcal{I}}(x, y)-\alpha(x, y)) \beta^{\prime}(x, y) & \forall\{x, y\} \in \mathrm{U} \subset \mathrm{S} \\ \hat{\mathcal{I}}(x, y)-\alpha(x, y)\left(1-\beta^{\prime \prime}(x, y)\right) & \forall\{x, y\} \in \mathrm{P} \subset \mathrm{S}\end{cases}$

It can be noted that $\mathcal{P}_{\ell}\left(\mathcal{I}_{s} \mid \alpha, \beta\right)$ models the error caused by the estimated parameters and encourages the recovered pixel values $\left(\hat{\mathcal{I}}_{s}(x, y)\right)$ to lie close to $\left(\mathcal{I}_{s}(x, y)\right)$ with variance $\sigma_{\mathcal{I}}^{2}$ following a Gaussian distribution. However, in the above formulation, there are nine unknowns for each pixel located inside the shadowed region. If we had a smaller scale problem (e.g., finding the precise shadow matte in the penumbra region by Chuang et al. [36]), we could have directly solved for the unknowns. But in our case, the large number of variables makes the likelihood calculation rather difficult and time consuming, especially when the number of shadowed pixels is large. We therefore resort to optimize the crude shadow-less image $(\hat{\mathcal{I}}(x, y))$ calculated in Sec. 4.1, Eq. 14.

The prior $\mathcal{P}_{\ell}(\beta)$ can be modeled as a Gaussian probability distribution centered at the mean $(\bar{\beta})$ of the 
neighboring pixels. This helps in estimating a smoothly varying beta mask. So,

$$
\mathcal{P}_{\ell}(\beta)=-\sum_{\{x, y\}} \frac{\left|\beta(x, y)-\bar{\beta}\left(x^{\prime}, y^{\prime}\right)\right|^{2}}{2 \sigma_{\beta}^{2}}, \quad\left(x^{\prime}, y^{\prime}\right) \in \mathcal{N}(x, y)
$$

The prior $\mathcal{P}_{\ell}(\alpha)$ can also be modeled in a similar fashion. However, we require $\alpha$ to model the variations in the penumbra region as well. Therefore, an additional term (called the 'image consistency term') is introduced in the prior $\mathcal{P}_{\ell}(\alpha)$ to smooth the estimated shadow-less image along the boundaries and to incorporate feedback from the previously estimated crude shadowless image. Therefore,

$$
\begin{aligned}
& \mathcal{P}_{\ell}(\alpha)=-\sum_{\{x, y\}} \frac{\left|\alpha(x, y)-\bar{\alpha}\left(x^{\prime}, y^{\prime}\right)\right|^{2}}{2 \sigma_{\alpha}^{2}}-\frac{1}{2 \sigma_{\mathcal{I}}^{2}} \\
& \sum_{\{x, y\} \in \mathrm{S}}\left(1-\frac{\lambda(x, y)}{\lambda_{\max }}\right)|\mathcal{I}(x, y)-\hat{\mathcal{I}}(x, y)|^{2}, \quad\left(x^{\prime}, y^{\prime}\right) \in \mathcal{N}(x, y)
\end{aligned}
$$

In the image consistency term (second term in Eq. 29), $\mathcal{I}(x, y)$ will take different values according to Eqs. 19 and 21:

$$
\mathcal{I}(x, y)= \begin{cases}\mathcal{I}_{u} / \beta^{\prime}(x, y)+\alpha(x, y) & \forall\{x, y\} \in \mathrm{U} \\ \mathcal{I}_{p}(x, y)+\alpha(x, y)\left(1-\beta^{\prime \prime}(x, y)\right) & \forall\{x, y\} \in \mathrm{P}\end{cases}
$$

\subsection{Parameter Estimation}

In spite of the crude shadow image estimation, it can be seen from Eq. 27 that the objective function is not linear or quadratic in term of the unknowns. To apply the gradient based energy optimization procedure, we simplify our problem by breaking it into two sub-optimization problems and apply an iterative joint optimization as follows:

For the umbra region,

$$
\beta^{\prime}(x, y)=\frac{\gamma_{\beta}^{2} \bar{\beta}\left(x^{\prime}, y^{\prime}\right)-\gamma_{\mathcal{I}}^{2}\left[\alpha(x, y) \mathcal{I}_{s}(x, y)-\hat{\mathcal{I}}(x, y) \mathcal{I}_{s}(x, y)\right]}{\gamma_{\beta}^{2}-\gamma_{\mathcal{I}}^{2}\left[2 \hat{\mathcal{I}}(x, y) \alpha(x, y)-\alpha^{2}(x, y)-\hat{\mathcal{I}}^{2}(x, y)\right]}
$$

For the penumbra:

$\beta^{\prime \prime}(x, y)=\frac{\alpha \gamma_{\mathcal{I}_{s}}^{2}[\Delta(x, y)+\alpha]+\gamma_{\beta}^{2} \bar{\beta}^{\prime \prime}+\alpha \gamma_{\mathcal{I}}^{2} \eta(x, y)[\Delta(x, y)+\alpha]}{\alpha^{2} \gamma_{\mathcal{I}_{s}}^{2}+\gamma_{\beta}^{2}+\alpha^{2} \gamma_{\mathcal{I}}^{2} \eta(x, y)}$

where, $\gamma=\sigma^{-1}$. To optimize $\alpha$, the parameter $\beta$ is held constant and the first order partial derivative is taken with respect to $\alpha$ and is set to zero. We get the following set of equations:

For the umbra region:

$$
\alpha(x, y)=\frac{\gamma_{\alpha}^{2} \bar{\alpha}\left(x^{\prime}, y^{\prime}\right)-\gamma_{\mathcal{I}}^{2}\left[\beta^{\prime}(x, y) \mathcal{I}_{s}(x, y)-\hat{\mathcal{I}}(x, y) \beta^{\prime 2}(x, y)\right]}{\gamma_{\alpha}^{2}+\gamma_{\mathcal{I}}^{2} \beta^{\prime 2}(x, y)}
$$

For the penumbra:

$\alpha(x, y)=\frac{-\gamma_{\mathcal{I}_{s}}^{2}\left(1-\beta^{\prime \prime}\right) \Delta(x, y)+\gamma_{\alpha}^{2} \bar{\alpha}-\gamma_{\mathcal{I}}^{2}\left(1-\beta^{\prime \prime}\right) \eta(x, y) \Delta(x, y)}{\gamma_{\mathcal{I}_{s}}^{2}\left(1-\beta^{\prime \prime}\right)^{2}+\gamma_{\alpha}^{2}+\gamma_{\mathcal{I}}^{2}\left(1-\beta^{\prime \prime}\right)^{2} \eta(x, y)}$

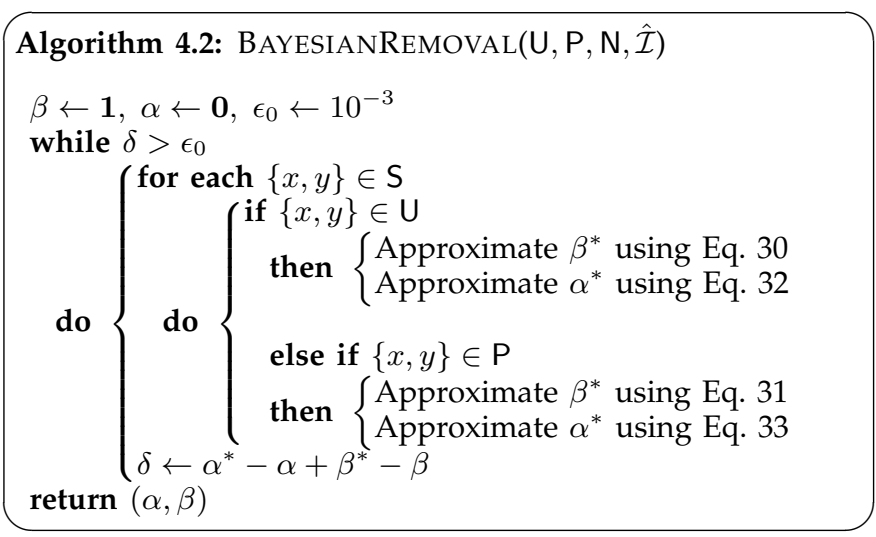

where, $\Delta(x, y)=\mathcal{I}_{s}(x, y)-\hat{\mathcal{I}}(x, y)$. We iteratively perform this procedure on each pixel in the shadow region until convergence.

\subsection{Boundary Enhancement in a Shadow-less Image}

The resulting shadow-less image exhibits traces of shadow boundaries in some cases. To remove these artifacts, we divide the shadow boundary into a group of segments, where each segment contains nearly similar colored pixels. The boundary segments which belong to the object shadow boundary are excluded from further processing. For each non-object shadow boundary segment, we perform Poisson smoothing [49] to conceal the shadow boundary artifacts.

\section{EXPERIMENTS AND ANALYSIS}

We evaluated our technique on three widely used and publicly available datasets. For the qualitative comparison of shadow removal, we also evaluate our technique on a set of commonly used images in the literature.

\subsection{Datasets}

UCF Shadow Dataset is a collection of 355 images together with their manually labeled ground truths. Zhu et al. have used a subset of 255/355 images for shadow detection [10].

CMU Shadow Dataset consists of 135 consumer grade images with labels for only those shadow edges which lie on the ground plane [11]. Since our algorithm is not restricted to ground shadows, we tested our approach on the more challenging criterion of full shadow detection which required the generation of new ground truths.

UIUC Shadow Dataset contains 108 images each of which is paired with its corresponding shadow-free image to generate a ground truth shadow mask [13].

Test/Train Split: For UCF and UIUC databases, we used the split mentioned in [10,13]. Since CMU database [11] did not report the split, we therefore used even/odd images for training/testing (following the procedure in Jiang et al. [12]). 


\begin{tabular}{|c|c|c|c|c|}
\hline & Methods & UCF Dataset & CMU Dataset & UIUC Dataset \\
\hline \multirow{7}{*}{ 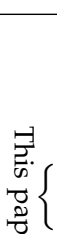 } & BDT-BCRF (Zhu et al. [10]) & $88.70 \%$ & - & - \\
\hline & BDT-CRF-Scene Layout (Lalonde et al. [11]) & - & $84.80 \%$ & - \\
\hline & Unary SVM-Pairwise (Guo et al. [13]) & $90.20 \%$ & - & $89.10 \%$ \\
\hline & Bright Channel-MRF (Panagopoulos et al. [15]) & $85.90 \%$ & _ & - \\
\hline & Illumination Maps-BDT-CRF (Jiang et al. [12]) & $83.50 \%$ & $84.98 \%$ & - \\
\hline & ConvNet(Boundary+Region) & $89.31 \%$ & $87.02 \%$ & $92.31 \%$ \\
\hline & ConvNet(Boundary+Region)-CRF & $\mathbf{9 0 . 6 5 \%}$ & $\mathbf{8 8 . 7 9} \%$ & $\mathbf{9 3 . 1 6} \%$ \\
\hline
\end{tabular}

TABLE 1: Evaluation of the proposed shadow detection scheme; All performances are reported in terms of pixel-wise accuracies.

\subsection{Evaluation of Shadow Detection}

\subsubsection{Results}

We assessed our approach both quantitatively and qualitatively on all the major datasets for single image shadow detection. We demonstrate the success of our shadow detection framework on different types of scenes including beaches, forests, street views, aerial images, road scenes and buildings. The databases also contain shadows under a variety of illumination conditions such as sunny, cloudy and dark environments. For quantitative evaluation, we report the performance of our framework when only the unary term (Eq. 3) was used for shadow detection. Further, we also report the perpixel accuracy achieved using the CRF model on all the datasets. This means that labels are predicted for every pixel in each test image and are compared with the ground-truth shadow masks. For the UCF and CMU datasets, the initial learning rate of $\eta_{0}=0.1$ was used, for the UIUC dataset we set $\eta_{0}=0.01$ based on the performance on a small validation set. After every 20 epochs the learning rate was decreased by a small factor $\beta=0.5$ which resulted in a best performance.

Table 1 summarizes the overall results of our framework and shows a comparison with several state-ofthe-art methods in shadow detection. It must be noted that the accuracy of Jiang's method [12] (on the CMU database) is given by the Equal Error Rate (EER). All other accuracies represent the highest detection rate achieved, which may not necessarily be an EER. Using the ConvNets and the CRF, we were able to get the best performance on the UCF, CMU and UIUC databases with a respective increase of $0.50 \%, 4.48 \%$ and $4.55 \%$ compared to the previous best results ${ }^{3}$. For the case of the UCF dataset, a gain of $0.5 \%$ accuracy may look modest. But it should be noted that the previous best methods of Zhu et al. [10] and Guo et al. [13] were only evaluated on a subset (255/355 images). In contrast, we report results on the complete dataset because the exact subset used in $[10,13]$ is not known. Compared to Jiang et al. [12], which is evaluated on the complete dataset, we achieved a relative accuracy gain of $8.56 \%$. On five sets of 255 randomly selected images from the UCF dataset, our method resulted in an accuracy of $91.4 \pm 4.2 \%$ which is a relative gain of $1.3 \%$ over Guo et al. [13].

Table 2 shows the comparison of class-wise accuracies. The true positives (correctly classified shadows)

3. Relative increase in performance is calculated by: $100 \times($ our accuracy - previous best)/previous best.

\begin{tabular}{|c|c|c|}
\hline Methods/Datasets & Shadows & Non-Shadows \\
\hline \multicolumn{3}{|l|}{ UCF Dataset } \\
\hline 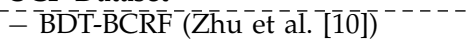 & $\overline{63 . \overline{9} \%}$ & $\overline{9} \overline{3} . \overline{4} \%$ \\
\hline - Unary-Pairwise (Guo et al. [13]) & $73.3 \%$ & $93.7 \%$ \\
\hline $\begin{aligned} \text { - Bright Channel-MRF } \\
\text { (Panagopoulos et al. [15]) }\end{aligned}$ & $68.3 \%$ & $89.4 \%$ \\
\hline - ConvNet(Boundary+Region) & $72.5 \%$ & $92.1 \%$ \\
\hline - ConvNet(Boundary+Region)-CRF & 78 . & $92.6 \%$ \\
\hline \multicolumn{3}{|l|}{ CMU Dataset } \\
\hline $\begin{array}{l}\text { BDT-CRF-Scene Layout } \\
\text { (Lalonde et al. [11]) }\end{array}$ & $73 . \overline{1} \%$ & $\overline{\mathbf{9}} \overline{\mathbf{6}} . \overline{4} \%$ \\
\hline - ConvNet(Boundary+Region) & $81.5 \%$ & $90.5 \%$ \\
\hline - ConvNet(Bou & $83.3 \%$ & $90.9 \%$ \\
\hline \multicolumn{3}{|l|}{ UIUC Dataset } \\
\hline - Ūnary-Pairwise $\overline{(C}$ & $\overline{71 . \overline{6} \%}$ & $\overline{9} \overline{5} . \overline{2} \%$ \\
\hline - ConvNet(Boundar & $83.6 \%$ & $94.7 \%$ \\
\hline - ConvNet(Boundary+Region)-CRF & $\mathbf{8 4 . 7} \%$ & $\mathbf{9 5 . 5} \%$ \\
\hline
\end{tabular}

TABLE 2: Class-wise accuracies of our proposed framework in comparison with the state-of-the-art techniques. Our approach gives the highest accuracy for the class 'shadows'.

are reported as the number of predicted shadow pixels which match with the ground-truth shadow mask. True negative (correctly classified non-shadows) are reported as the number of predicted non-shadow pixels which match with the ground-truth non-shadow mask. It is interesting to see that our framework has the highest shadow detection performance on the UCF, CMU and UIUC datasets. For the case of CMU dataset, our approach got a relatively lower non-shadow region detection accuracy of $90.9 \%$ compared to $96.4 \%$ of Lalonde et al. [11]. This is due to the reason that [11] only consider ground shadows and thus ignore many false negatives. In contrast, our method is evaluated on more challenging case of general shadow detection i.e. all types of shadows. The ROC curve comparisons are shown in Fig. 10. The plotted ROC curves represent the performance of the unary detector since we cannot generate ROC curves from the outcome of the CRF model. Our approach achieves the highest AUC measures for all datasets (Fig. 10).

Some representative qualitative results are shown in Fig. 9 and Fig. 11. The proposed framework successfully detects shadows in dark environments (Fig. 9: $1^{\text {st }}$ row, middle image) and distinguishes between dark nonshadow regions and shadow regions (Fig. 9: $2^{\text {nd }}$ row, $2^{\text {nd }}$ and $5^{\text {th }}$ image from left). It performs equally well on satellite images (Fig. 9: last column) and outdoor scenes with street views (Fig. 9: $1^{\text {st }}$ row, $3^{\text {rd }}$ and $5^{\text {th }}$ images; $2^{\text {nd }}$ row, middle image), buildings (Fig. 9: $1^{\text {st }}$ column) and shadows of animals and humans (Fig. 9: $2^{\text {nd }}$ column). 

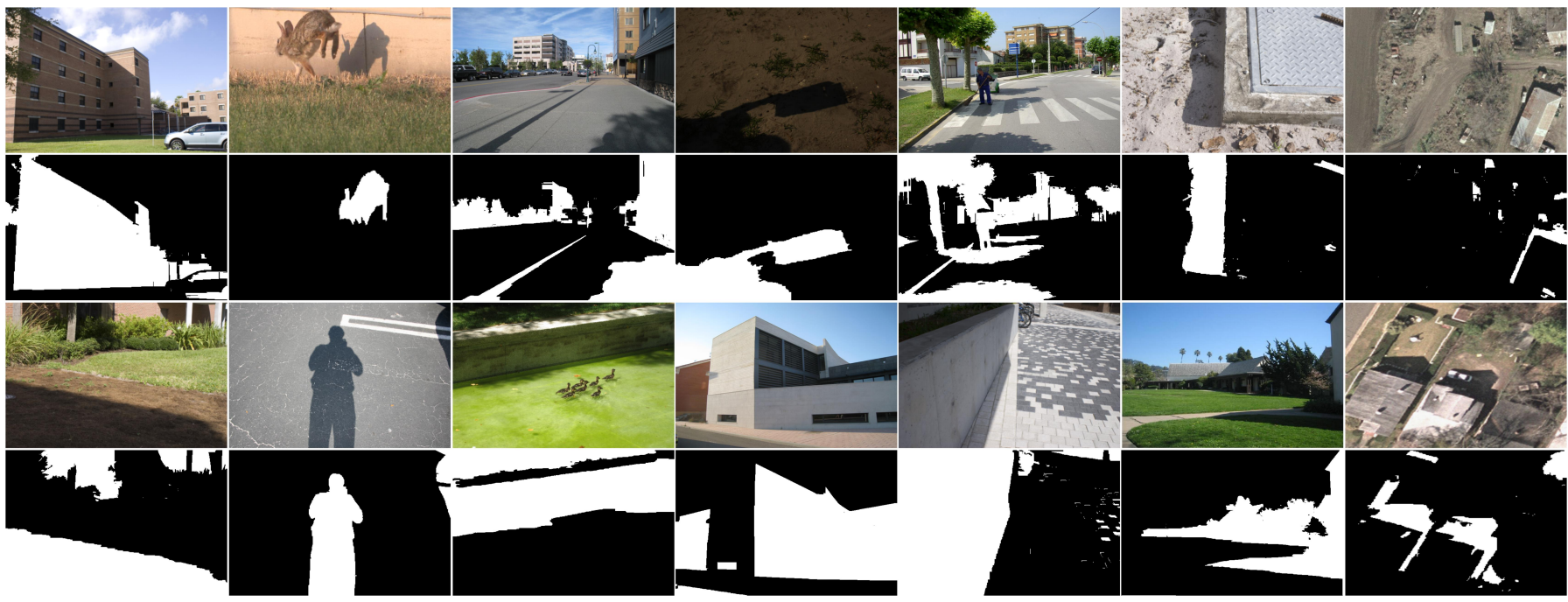

Fig. 9: Examples of our results; Images $\left(1^{\text {st }}, 3^{\text {rd }}\right.$ row $)$ and shadow masks $\left(2^{\text {nd }}, 4^{\text {th }}\right.$ row $)$; Shadows are in white.

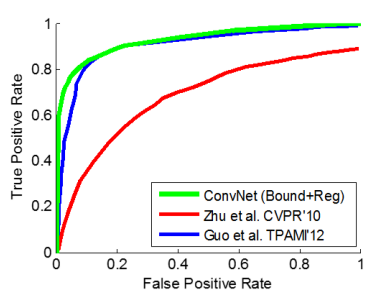

(a) UCF Shadow Dataset

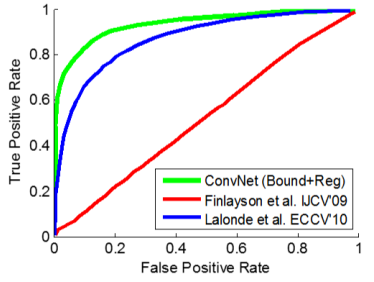

(b) CMU Shadow Dataset

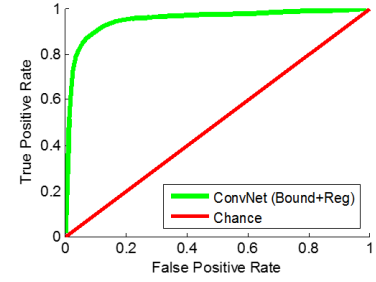

(c) UIUC Shadow Dataset

Fig. 10: ROC curve comparisons of proposed framework with previous works.

\begin{tabular}{l||ccc}
\multicolumn{1}{c||}{ Tested on } & \multicolumn{3}{c}{ Trained on } \\
& UCF & CMU & UIUC \\
\hline UCF & - & $80.3 \%$ & $80.5 \%$ \\
CMU & $77.7 \%$ & - & $76.8 \%$ \\
UIUC & $82.8 \%$ & $81.5 \%$ & -
\end{tabular}

TABLE 3: Results when ConvNets were trained and tested across different datasets.

\subsubsection{Discussion}

The previously proposed methods (e.g., Zhu et al. [10], Lalonde et al. [11]) that use a large number of handcrafted features, not only require a lot of effort in their design but also require long training times when ensemble learning methods are used for feature selection. As an example, Zhu et al. [10] extracted different shadow variant and invariant features alongside an additional 40 classification results from the Boosted Decision Tree (BDT) for each pixel as their features. Their approach required a huge amount of memory ( $\sim$ GB for 125 training images of average size of approximately $480 \times 320$ ). Even after parallelization and training on multiple processors, they reported 10 hours of training with 125 images. Lalonde et al. [11] used 48 dimensional feature vectors extracted at each pixel and fed these to a boosted decision tree in a similar manner as Zhu et al. [10]. Jiang et al. included illumination features on top of the features that are used by Lalonde et al. [11]. Although, enriching the feature set in this manner improves the performance, it not only takes much more effort to design such features but it also slows down the detection procedure. In contrast, our feature learning procedure is fully automatic and requires only $\sim 1 \mathrm{~GB}$ memory and approximately one hour training for each of the UCF, CMU and UIUC databases. The proposed approach is also efficient at test time because the ConvNet feature extraction and unary potential computation take an average of $1.3 \pm 0.35$ sec per image on the UCF, CMU and UIUC databases.

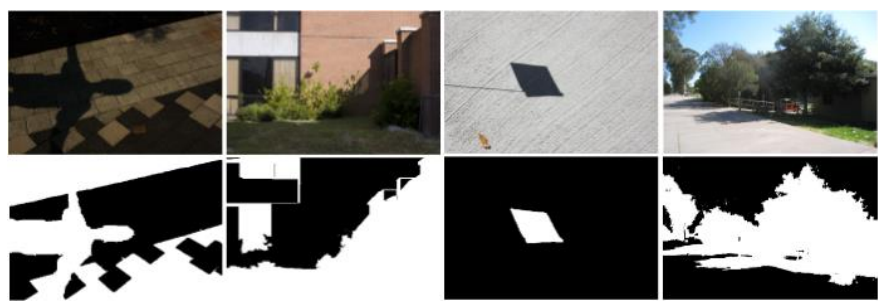

Fig. 11: Examples of Ambiguous Cases: (From left to right) Our framework misclassified a dark non-shadow region, textureless black window glass, very thin shadow region and trees due to complex self shading patterns. (Best viewed in color)

The graph-cut inference step used for the CRF energy minimization is also fast and takes $0.21 \pm 0.03 \mathrm{sec}$ per image on average. Overall, our technique takes $2.8 \pm 0.81$ sec per image for shadow detection. In comparison, the method by Guo et al. [13] takes $40.05 \pm 10 \mathrm{sec}$ per image for shadow detection.

We extensively evaluated our approach on all available databases and our proposed framework turned out to be fairly generic and robust to variations. It achieved the best results on all the single image shadow databases known to us. In contrast, previous techniques were only tested on a portion of database [11], one [10] or at most two databases [13]. Another interesting observation was that the proposed framework performed reasonably well when our ConvNets were trained on one dataset and tested on another dataset. Table 3 summarizes the results of cross-dataset evaluation experiments. These 

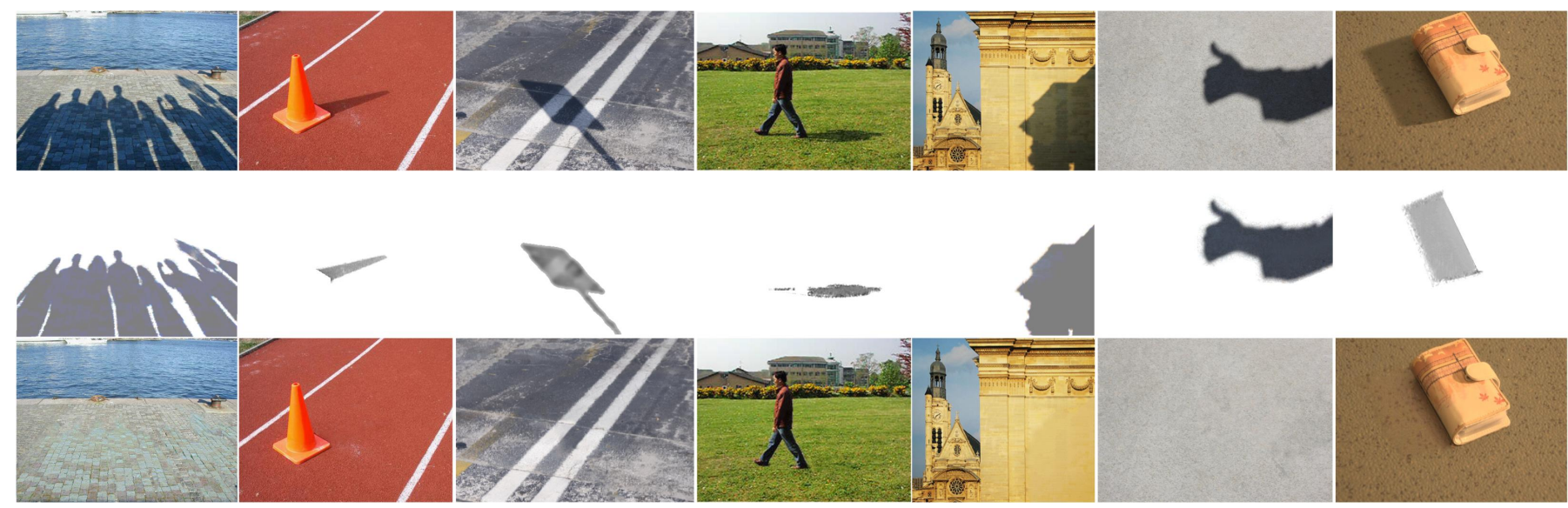

Fig. 12: Qualitative Evaluation: Shadow recovery on sample images from UIUC, UCF databases and other images used in literature. Given a original image with shadow mask (first row), our method is able to extract exact shadows (second row) and to automatically recover the shadow-less images (third row). (Best viewed in color)

performance levels show that the feature representations learned by the ConvNets across the different datasets were common to a large extent. This observation further supports our claim regarding the generalization ability of the proposed framework.

In our experiments, objects with dark albedo turned out to be a difficult case for shadow detection. Moreover, some ambiguities were caused by the complex self shading patterns created by tree leaves. There were some inconsistencies in the manually labeled ground-truths, in which a shadow mask was sometimes missing for an attached shadow. Narrow shadowy regions caused by structures like poles and pipes also proved to be a challenging case for shadow detection. Examples of the above mentioned failure cases are shown in Fig. 11.

\subsection{Evaluation of Shadow Removal}

For a quantitative evaluation of our shadow removal framework, we used all images from the UIUC Shadow dataset which come with their corresponding shadowfree ground truths [13]. The qualitative results of our method are evaluated against the common evaluation images used in the literature for a fair comparison. To further illustrate the performance of our algorithm, we also included qualitative results on some example images from UIUC, UCF and CMU shadow datasets.

\subsubsection{Quantitative Evaluation}

Table 4 presents the per pixel root mean square error (RMSE) for the UIUC dataset, calculated in LAB color space [13]. The first row gives the actual error between the same image, with and without shadow. The difference between the two versions of the same image is calculated for both the shadow and the lit regions. Note that the error is large for the shadowed region (as expected), but it is not zero for the lit regions for two reasons: the shadow masks are not perfect and there is a little difference in the light intensity due to the change in the ambient light for the lit regions when

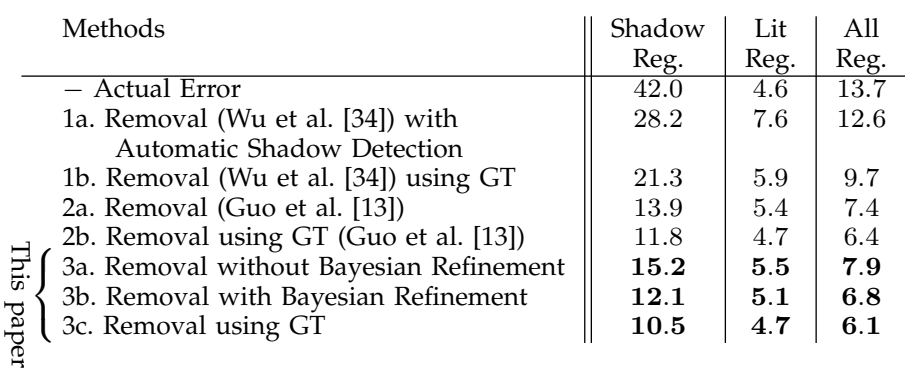

TABLE 4: Quantitative Evaluation: RMSE per pixel for the UIUC Subset of Images. (The smaller RMSE the better)

the object casting shadow is present. We achieved an average RMSE error of 6.8 compared to 7.4 and 12.6 achieved by the methods of Guo et al. [13] and Wu et al. [34], respectively. Following Guo et al. [13], we also include the removal performance when the ground truth (GT) shadow masks are used for removal. This gives a more precise estimate of the performance of the recovery algorithm. When we evaluated our method using GT masks, our method achieved an error of 6.1 compared to 6.4 and 9.7 reported by [13] and [34] respectively. We also tested the removal results without the Bayesian optimization, which resulted in an RMSE error of 7.9. This is high compared to the results achieved after optimization. In summary, our method achieved a reduction in error of $8.1 \%$ (removal using the detected masks) and $4.6 \%$ (removal using ground truths) compared to the approach of Guo et al. in [13].

\subsubsection{Qualitative Evaluation}

For the qualitative evaluation, we show some example images and their corresponding recovered images along with the shadow masks in Fig. 12. It can be seen that our method works well under different settings e.g., outdoor images (first five images from the left) and indoor images (first two images from the right). The complex texture in the shadow regions is preserved and the arbitrary shadow matte are precisely recovered. Note that while 


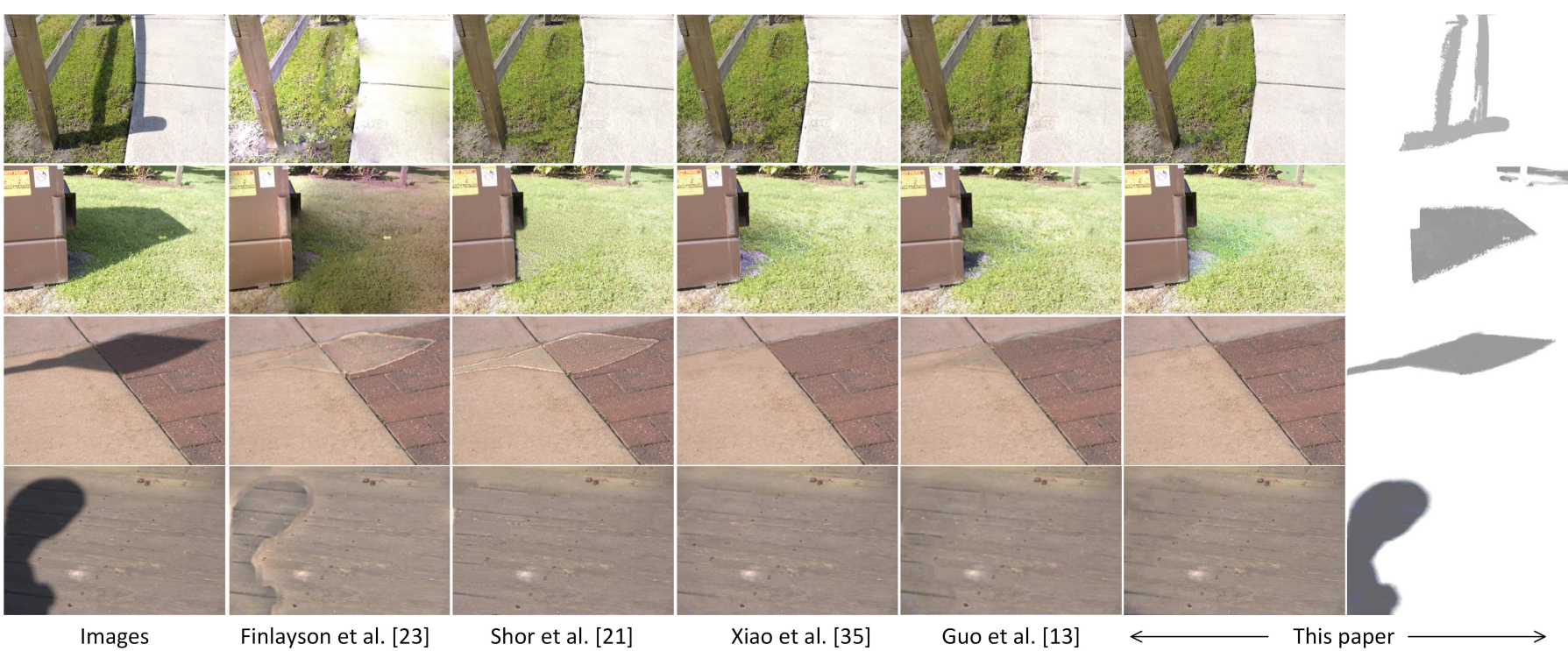

Fig. 13: Comparison with Automatic/Semi-Automatic Methods: Recovered shadow-less images are compared with the state-ofthe-art shadow removal methods which are either automatic [13, 23] or require minimal user input [21, 35]. We compare our work with: (from left to right) Finlayson et al. [23], Shor and Lischinski [21], Xiao et al. [35] and Guo et al. [13] respectively. The results achieved using our method (second column from right) are comparable or better than the previous best results (columns 1-5 from left). Additionally, our method works without any user input and provides shadow matte (last column) which can be used to generate composite images. (Best viewed in color and enlarged)

our method can remove hard and smooth shadows (e.g., $1^{\text {st }}, 5^{\text {th }}$ and $6^{\text {th }}$ image from left), it also works well for the soft and variable shadows (e.g., $2^{\text {nd }}, 3^{\text {rd }}$ and $4^{\text {th }}$ image from left). Overall, the results are visually pleasing and the extracted shadow matte are smooth and accurate.

\subsubsection{Comparisons}

We provide a qualitative comparison with two distinct categories of shadow removal methods. First, we show comparisons (see Fig. 13) with the state-of-theart shadow removal methods which are either fully automatic (e.g., [13, 23]) or require minimal user input (e.g., [21, 35]). From left to right we show the original image along with the results from Finlayson et al. [23], Shor and Lischinski [21], Xiao et al. [35], Guo et al. [13] and our technique. In comparison to the previous automatic and semi-automatic (requiring minimal user input) methods, our approach produces cleaner recovered images (second column from the right) along with an accurate shadow matte (right most column).

Since, there are only very few automatic shadow removal methods in the literature, we also compare our approach with the most popular approaches but which require user input (see Fig. 14). From left to right, we show our recovered images (bottom row) along with the results from $\mathrm{Wu}$ et al. [34], Liu and Gleicher [27], Arbel and Hel-Or [29], Vicente and Samaras [50], Fredembach and Finlayson [26] and Kwatra et al. [30]. For the 'puzzled child' image, it can be seen that the contrast of the recovered region is much better than the one recovered by $\mathrm{Wu}$ et al. [34]. The shadow-less image has no trace of strong shadow boundaries and the recovery in the penumbra region is smooth due to introduction of $\alpha$ in the model and the exclusion of the spatial affinity term [34] or boundary nullification [33] during the rough shadowless image estimation process. Similar effects can be seen with the other images; e.g., in $3^{\text {rd }}$ image from the left, the result of Arbel and Hel-Or [29] has a high contrast while our result is smooth and successfully retains texture. Similarly, for the case of the $4^{t h}, 5^{t h}$ and $6^{t h}$ images from the left, our shadow removal result is visually pleasing and considerably better than the recent state-of-the-art methods. Note however that the recovery result of the $2^{\text {nd }}$ image from the left has an over-smoothing effect, probably because the color distributions of differently colored shadowed regions could not be separated during the Gaussian fitting process. Overall, the results are quite reasonable considering that the algorithm does not require any user assistance and it does not make any prior assumptions such as a Planckian light source or a narrow-band camera.

\subsubsection{Failure Cases and Limitations}

Our shadow removal technique does not perform well on curved surfaces and in the case of highly non-uniform shadows (e.g., Fig. 15: $1^{s t}$ and $3^{\text {rd }}$ image from left). Since, we apply a multi-level color transfer scheme, very fine texture details of image regions with similar appearance can be removed during this transfer process (e.g., Fig. 15: $2^{\text {nd }}$ image from left). For the cases of shadows in dark environments, our method appears to increase the contrast of the recovered region. These limitations are due to the constraints imposed on the shadow generation model, where the higher order statistics are ignored during the shadow generation process (Eqs. 19 and 21). 

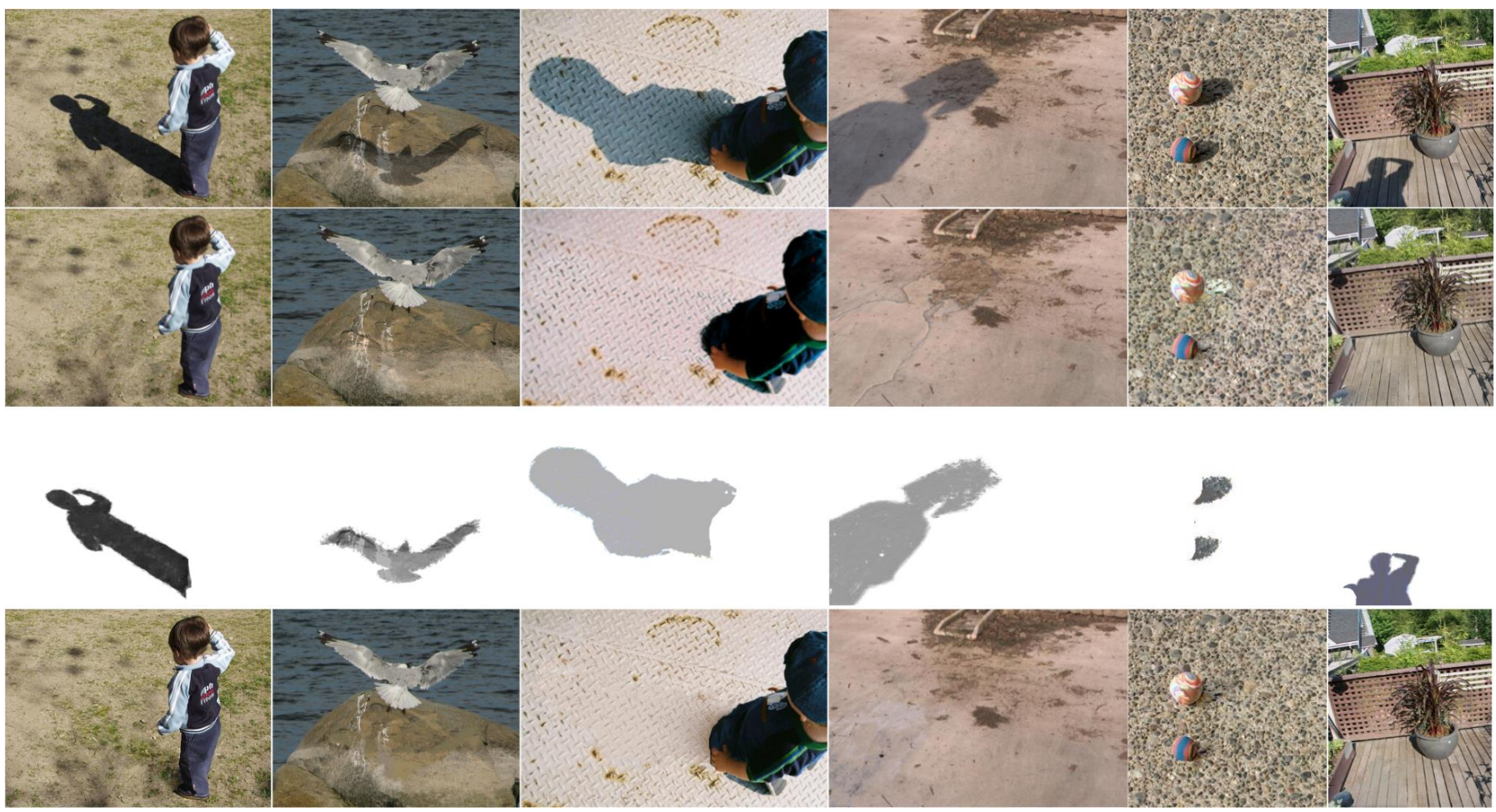

Fig. 14: Comparison with Methods Requiring User Interaction: Recovered shadow-less images are compared with the state-ofthe-art shadow removal methods (which require considerable amount of user input). We compare our work with: (from left to right in the second row) Wu et al. [34], Liu and Gleicher [27], Arbel and Hel-Or [29], Vicente and Samaras [50], Fredembach and Finlayson [26] and Kwatra et al. [30] respectively. The results achieved by our method (last row) are comparable or better than the previous best results (second row). Additionally, our method works without any user input and provides shadow matte (third row) which can be used to generate composite images. (Best viewed in color and enlarged)

\subsubsection{Discussion}

Our method does not require any user input and it automatically removes shadow after its detection. The proposed shadow removal approach makes comparatively fewer assumptions about the scene type, the type of light source or camera. The only assumptions are that of Lambertian surfaces and the correspondence between the shadow and the non-shadow region color distributions. The shadow removal method of [33, 34] cannot separate the shadow from shading. With the inclusion of the image consistency term in $\mathcal{P}_{\ell}\left(\mathcal{I}_{s} \mid \alpha, \beta\right)$, we are able to deal with the shading by introducing a penalty on the distribution of the shadow effect through the parameters $\beta$ and $\alpha$. The proposed shadow removal approach takes $82.2 \pm 25 \mathrm{sec}$ for each image on the UIUC database. The main overhead during the shadow removal process is the Bayesian refinement step (which is required mainly for shadow matting). It takes $73.6 \pm 20 \mathrm{sec}$ out of $82.2 \pm 25$ sec per image on the UIUC database. In comparison, the method by Guo et al. [13] takes $104.7 \pm 18 \mathrm{sec}$ for shadow removal. The main overhead in their removal process is also due to Levin et al.'s matting algorithm [51] which takes around $91.4 \pm 11 \mathrm{sec}$ per image.

\subsection{Applications}

Shadow detection, removal and matting have a number of applications. A direct application is the generation

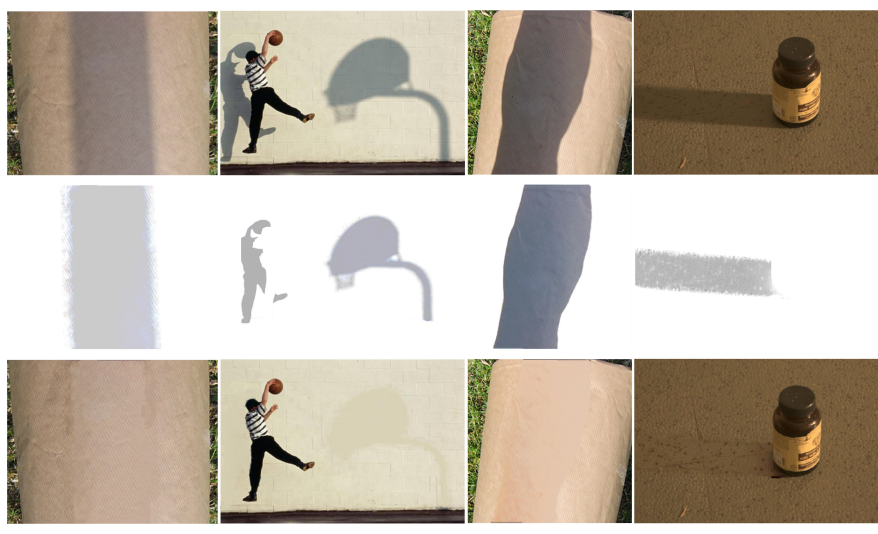

Fig. 15: Examples of Failure Cases: Our technique does not perfectly remove shadows on curved surfaces, highly nonuniform shadows and shadows in dark environments. (Best viewed in color and enlarged)

of visually appealing photographs and the removal of unwanted shadows. Some other applications include:

Shadow Compositing: Fig. 16(a) shows examples of shadow compositing. The extracted shadow matte can be used to depict a realistic image compositing. For example, the first image from the left did not originally contain the flying bird and its shadow. If we had added just the bird, it would have looked unrealistic. With the addition of a texture-free shadow matte, the photograph looks natural and realistic. In the remaining three im- 


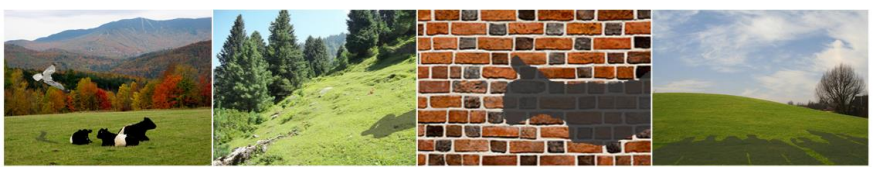

(a) Shadow Compositing

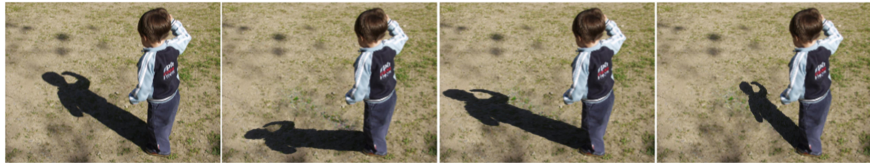

(b) Image Editing

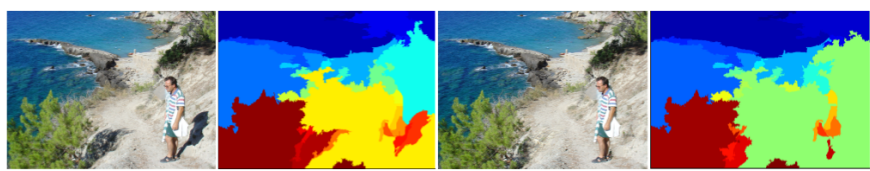

(c) Image Parsing
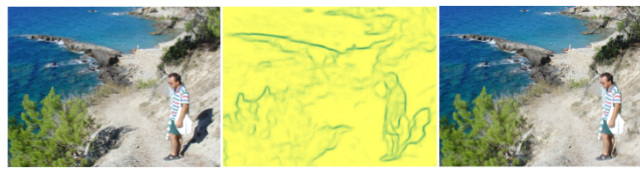

(d) Boundary Detection

Fig. 16: Different Applications of Shadow Detection, Removal and Matting. (Best viewed in color and enlarged)

ages, we combine extracted shadows with the original images to create fake effects.

Image Editing: Fig. 16(b) shows how a detected shadow can be edited to create fake effects. For example, shadow direction/length can be modified to give a fake impression of illumination source or time of day.

Image Parsing: Fig. 16(c) shows how shadow removal can increase the accuracy of segmentation methods (e.g., $[52,53])$. The segmentations are computed using the graph based technique of [46] (we used a minimum region size of 600 ). It can be seen that shadows change the appearance of a class (e.g., ground in this case) and thus can introduce errors in the segmentation process.

Boundary Detection: We tested a recently proposed boundary detector [54] on the original and recovered image (Fig. 16(d)). The boundaries identified in the recovered image are more accurate. Since shadows do not constitute an object class, the recovered image can help in achieving more accurate object detection proposals and consequently a higher recognition performance.

\section{Conclusion}

We presented a data-driven approach to learn the most relevant features for the detection of shadows from a single image. We demonstrated that our framework performs the best on a number of databases regardless of the shape of objects casting shadows, the environment and the type of scene. We also proposed a shadow removal framework which extracts the shadow matte along with the recovered image. A Bayesian formulation constitutes the basis of our shadow removal procedure and thereby makes use of an improved shadow generation model. Our shadow detection results show that a combination of boundary and region ConvNets incorporated in the CRF model provides the best performance. For shadow removal, the multi-level color transfer followed by the Bayesian refinement performs well on unconstrained images. The proposed framework has a number of applications including image editing and enhancement tasks.

\section{ACKNOWLEDGMENTS}

The authors would like to thank the anonymous reviewers for their valuable comments and suggestions to improve the quality of the paper. This research was supported by the IPRS awarded by The University of Western Australia and the Australian Research Council (ARC) grants DP110102166, DP150100294 and DE120102960. The Flickr images are used under a Creative Commons license from Flickr users: mountaintrekker2001, Ulrich J and Kanshian.

\section{REFERENCES}

[1] L. Da Vinci, Notebooks. Oxford University Press, 2008.

[2] Y. Matsushita, K. Nishino, K. Ikeuchi, and M. Sakauchi, "Illumination normalization with time-dependent intrinsic images for video surveillance," TPAMI, vol. 26, no. 10, pp. 1336-1347, 2004.

[3] T. Okabe, I. Sato, and Y. Sato, "Attached shadow coding: estimating surface normals from shadows under unknown reflectance and lighting conditions," in ICCV. IEEE, 2009, pp. 1693-1700.

[4] D. Kersten, D. Knill, P. Mamassian, and I. Bülthoff, "Illusory motion from shadows." Nature, vol. 379, no. 6560, p. 31, 1996.

[5] I. Sato, Y. Sato, and K. Ikeuchi, "Illumination from shadows," TPAMI, vol. 25, no. 3, pp. 290-300, 2003.

[6] A. Prati, I. Mikic et al., "Detecting moving shadows: Algorithms and evaluation," TPAMI, vol. 25, pp. 918-923, 2003.

[7] M. Hayat, M. Bennamoun, and S. An, "Deep reconstruction models for image set classification," Pattern Analysis and Machine Intelligence, IEEE Transactions on, vol. 37, no. 4, pp. 713-727, April 2015.

[8] D. H. Hubel and T. N. Wiesel, "Receptive fields, binocular interaction and functional architecture in the cat's visual cortex," The Journal of Physiology, vol. 160, no. 1, p. 106, 1962

[9] S. H. Khan, M. Bennamoun et al., "Automatic feature learning for robust shadow detection," in CVPR, 2014.

[10] J. Zhu, K. G. Samuel, S. Z. Masood, and M. F. Tappen, "Learning to recognize shadows in monochromatic natural images," in CVPR, 2010, pp. 223-230.

[11] J.-F. Lalonde, A. A. Efros, and S. G. Narasimhan, "Detecting ground shadows in outdoor consumer photographs," in ECCV. Springer, 2010, pp. 322-335.

[12] X. Jiang, A. J. Schofield, and J. L. Wyatt, "Shadow detection based on colour segmentation and estimated illumination." in BMVC, 2011, pp. 1-11.

[13] R. Guo, Q. Dai, and D. Hoiem, "Paired regions for shadow detection and removal," TPAMI, 2012.

[14] E. Salvador, A. Cavallaro, and T. Ebrahimi, "Cast shadow segmentation using invariant color features," CVIU, vol. 95, no. 2, pp. 238-259, 2004.

[15] R. Panagopoulos, C. Wang, and D. Samaras, "Estimating shadows with the bright channel cue," in CRICV (with ECCV). Citeseer, 2010.

[16] E. Vazquez, R. Baldrich et al., "Describing reflectances for color segmentation robust to shadows, highlights, and textures," TPAMI, vol. 33, no. 5, pp. 917-930, 2011. 
[17] A. Panagopoulos, C. Wang et al., "Illumination estimation and cast shadow detection through a higher-order graphical model," in CVPR, 2011, pp. 673-680.

[18] G. Finlayson, C. Fredembach, and M. S. Drew, "Detecting illumination in images," in ICCV. IEEE, 2007, pp. 1-8.

[19] A. J. Joshi and N. P. Papanikolopoulos, "Learning to detect moving shadows in dynamic environments," TPAMI, vol. 30, no. 11, pp. 2055-2063, 2008.

[20] I. Huerta, M. Holte et al., "Detection and removal of chromatic moving shadows in surveillance scenarios," in ICCV. IEEE, 2009, pp. 1499-1506.

[21] Y. Shor and D. Lischinski, "The shadow meets the mask: Pyramid-based shadow removal," in Computer Graphics Forum, vol. 27, no. 2. Wiley, 2008, pp. 577-586.

[22] A. Bousseau, S. Paris, and F. Durand, "User-assisted intrinsic images," in TOG, vol. 28, no. 5. ACM, 2009.

[23] G. D. Finlayson, S. D. Hordley, C. Lu, and M. S. Drew, "On the removal of shadows from images," TPAMI, vol. 28, no. 1, pp. 59-68, 2006.

[24] G. D. Finlayson, S. D. Hordley, and M. S. Drew, "Removing shadows from images," in ECCV, vol. 2353. Springer, 2002, pp. 823-836.

[25] G. D. Finlayson, M. S. Drew, and C. Lu, "Entropy minimization for shadow removal," IJCV, vol. 85, no. 1, pp. 35-57, 2009.

[26] C. Fredembach and G. D. Finlayson, "Hamiltonian path based shadow removal." in BMVC, 2005, pp. 502-511.

[27] F. Liu and M. Gleicher, "Texture-consistent shadow removal," in ECCV. Springer, 2008, pp. 437-450.

[28] A. Mohan, J. Tumblin, and P. Choudhury, "Editing soft shadows in a digital photograph," Computer Graphics and Applications, IEEE, vol. 27, no. 2, pp. 23-31, 2007.

[29] E. Arbel and H. Hel-Or, "Shadow removal using intensity surfaces and texture anchor points," TPAMI, vol. 33, no. 6, pp. 1202-1216, 2011.

[30] V. Kwatra, M. Han, and S. Dai, "Shadow removal for aerial imagery by information theoretic intrinsic image analysis," in ICCP. IEEE, 2012, pp. 1-8.

[31] Q. Yang, K.-H. Tan, and N. Ahuja, "Shadow removal using bilateral filtering," TIP, vol. 21, pp. 4361-4368, 2012.

[32] E. Reinhard, M. Adhikhmin, B. Gooch, and P. Shirley, "Color transfer between images," Computer Graphics and Applications, IEEE, vol. 21, no. 5, pp. 34-41, 2001.

[33] T.-P. Wu and C.-K. Tang, "A bayesian approach for shadow extraction from a single image," in ICCV, vol. 1. IEEE, 2005, pp. 480-487.

[34] T.-P. Wu, C.-K. Tang et al., "Natural shadow matting," ACM TOG, vol. 26, no. 2, p. 8, 2007.

[35] C. Xiao, R. She et al., "Fast shadow removal using adaptive multi-scale illumination transfer," in Computer Graphics Forum, vol. 32, no. 8. Wiley, 2013, pp. 207-218.

[36] Y.-Y. Chuang, B. Curless et al., "A bayesian approach to digital matting," in CVPR, vol. 2. IEEE, 2001, pp. II-264.

[37] A. Levin and Y. Weiss, "User assisted separation of reflections from a single image using a sparsity prior," TPAMI, vol. 29, no. 9, pp. 1647-1654, 2007.

[38] Y.-F. Su and H. H. Chen, "A three-stage approach to shadow field estimation from partial boundary information," TIP, vol. 19, no. 10, pp. 2749-2760, 2010.

[39] R. Achanta, A. Shaji et al., "Slic superpixels compared to state-of-the-art superpixel methods," TPAMI, vol. 34, no. 11, pp. 2274-2282, 2012.

[40] P. Arbelaez, M. Maire, C. Fowlkes, and J. Malik, "Contour detection and hierarchical image segmentation," TPAMI, vol. 33, no. 5, pp. 898-916, 2011.

[41] N. V. Chawla, K. W. Bowyer, L. O. Hall, and W. P. Kegelmeyer, "Smote: synthetic minority over-sampling technique," Journal of AI Research, vol. 16, no. 1, pp. 321357,2002
[42] D. Ciresan, U. Meier, and J. Schmidhuber, "Multi-column deep neural networks for image classification," in CVPR, 2012, pp. 3642-3649.

[43] Y. LeCun, L. Bottou, G. Orr, and K.-R. Mller, "Efficient backprop," in Neural Networks: Tricks of the Trade, ser. LNCS. Springer, 2012, vol. 7700, pp. 9-48.

[44] M. Szummer, P. Kohli, and D. Hoiem, "Learning crfs using graph cuts," in ECCV. Springer, 2008, pp. 582-595.

[45] Y. Boykov and G. Funka-Lea, "Graph cuts and efficient nd image segmentation," IJCV, vol. 70, pp. 109-131, 2006.

[46] P. F. Felzenszwalb and D. P. Huttenlocher, "Efficient graph-based image segmentation," IJCV, vol. 59, no. 2, pp. 167-181, 2004.

[47] A. Criminisi, P. Pérez, and K. Toyama, "Region filling and object removal by exemplar-based image inpainting," TIP, vol. 13, no. 9, pp. 1200-1212, 2004.

[48] H. Barrow and J. Tenenbaum, "Recovering intrinsic scene characteristics," Computer vision systems, p. 2, 1978.

[49] P. Pérez, M. Gangnet, and A. Blake, "Poisson image editing," in ACM Transactions on Graphics (TOG), vol. 22, no. 3. ACM, 2003, pp. 313-318.

[50] T. Vicente and D. Samaras, "Single image shadow removal via neighbor based region relighting," CPCV (with ECCV), 2014.

[51] A. Levin, D. Lischinski, and Y. Weiss, "A closed-form solution to natural image matting," TPAMI, vol. 30, no. 2, pp. 228-242, 2008.

[52] S. H. Khan, M. Bennamoun, F. Sohel, and R. Togneri, "Geometry driven semantic labeling of indoor scenes," in Computer Vision-ECCV 2014. Springer, 2014, pp. 679-694.

[53] S. H. Khan, X. He, M. Bennamoun, F. Sohel, and R. Togneri, "Separating objects and clutter in indoor scenes," CPPR, 2015.

[54] P. Dollár and C. L. Zitnick, "Structured forests for fast edge detection," in ICCV. IEEE, 2013.

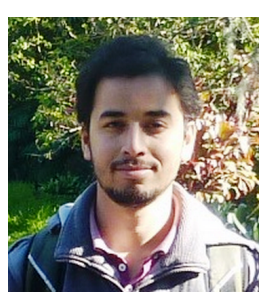

Salman H. Khan received his B.E. degree in Electrical Engineering from National University of Science and Technology (NUST), Pakistan in 2012. During 2010-12, he remained associated with Research Laboratory of Communications, Networks and Multimedia (CoNNekT), SEECS, NUST and Next Generation Intelligent Networks Research Center (nexGIN RC), FAST-NUCES, Islamabad. He is currently a $\mathrm{PhD}$ candidate at the University of Western Australia (UWA) where he was awarded prestigious International Postgraduate Research Scholarship (IPRS) for doctoral studies. His research interests include computer vision and pattern recognition.

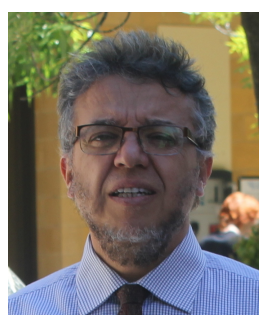

Mohammed Bennamoun received his MSc degree in control theory from Queens University, Kingston, Canada, and his Ph.D. degree in computer vision from Queens/QUT in Brisbane, Australia. He is currently a Winthrop Professor at the University of Western Australia, Australia. He served as a guest editor for a couple of special issues in International journals such as the International Journal of Pattern Recognition and Artificial Intelligence (IJPRAI). He was selected to give conference tutorials at the European Conference on Computer Vision (ECCV) and the International Conference on Acoustics Speech and Signal Processing (ICASSP). He organized several special sessions for conferences, e.g., the IEEE International Conference in Image Processing (ICIP). He also contributed in the organization of many local and international conferences. His research interests include control theory, robotics, object recognition, artificial neural networks, signal/image processing, and computer vision. $\mathrm{He}$ published more than 300 journal and conference publications. 
This is the author's version of an article that has been published in this journal. Changes were made to this version by the publisher prior to publication.

The final version of record is available at http://dx.doi.org/10.1109/TPAMI.2015.2462355

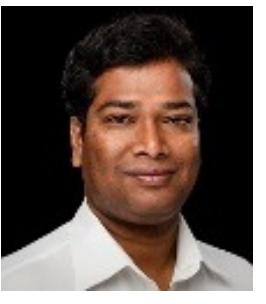

Ferdous A Sohel received PhD degree majoring Image Processing from Monash University, Australia (in 2009). He joined the School of Computer Science and Software Engineering, The University of Western Australia in 2008 where he is currently a Research Assistant Professor. His research interests include computer vision, image processing, pattern recognition, and video coding. He has published more than 50 scientific articles. He is a recipient of prestigious Discovery Early Career Research Award (DECRA) funded by the Australian Research Council. He is a recipient of the Early Career Investigator award (UWA) and the best PhD thesis medal form Monash University.

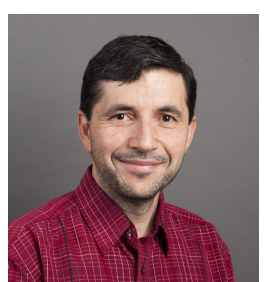

Roberto Togneri (M89-SM04) received the B.E. degree in 1985, and the Ph.D degree in 1989 both from the University of Western Australia. $\mathrm{He}$ joined the School of Electrical, Electronic and Computer Engineering at The University of Western Australia in 1988, where he is now currently a Professor. Prof Togneri is a member of the Signals and Systems Engineering Research Group and heads the Signal and Information Processing Lab. His research activities include signal processing and robust feature extraction of speech signals, statistical and neural network models for speech and speaker recognition, audio-visual recognition and biometrics, and related aspects of communications, information retrieval, and pattern recognition. He has published over 150 refereed journal and conference papers in the areas of signals and information systems, was the chief investigator on two Australian Research Council Discovery Project research grants from 2010 to 2013, and is currently an Associate Editor for IEEE Signal Processing Magazine Lecture Notes and Editor for IEEE Transactions on Speech, Audio and Language Processing. 